Acta Crystallographica Section A

Foundations of Crystallography

ISSN 0108-7673

Received 3 May 2004

Accepted 16 August 2004

(C) 2005 International Union of Crystallography Printed in Great Britain - all rights reserved

\section{Ferroelastic phase transitions: structure and micro- structure}

\author{
Ekhard K. H. Salje, ${ }^{a *}$ Stuart A. Hayward ${ }^{a}$ and William T. Lee ${ }^{a, b}$ \\ ${ }^{a}$ Department of Earth Sciences, University of Cambridge, Downing Street, Cambridge CB2 3EQ, \\ England, and ${ }^{\mathbf{b}}$ Mineralogisch-Petrographisches Institut, Universität Hamburg, Germany. Corre- \\ spondence e-mail: es10002@esc.cam.ac.uk
}

Landau-type theories describe the observed behaviour of phase transitions in ferroelastic and co-elastic minerals and materials with a high degree of accuracy. In this review, the derivation of the Landau potential $G=\frac{1}{2} A \theta_{S}\left[\operatorname{coth}\left(\theta_{\mathrm{S}} / T\right)-\right.$ $\left.\operatorname{coth}\left(\theta_{S} / T_{C}\right)\right] Q^{2}+\frac{1}{4} B Q^{4}+\ldots$ is derived as a solution of the general $\phi^{4}$ model. The coupling between the order parameter and spontaneous strain of a phase transition brings the behaviour of many phase transitions to the mean-field limit, even when the atomistic mechanism of the transition is spin-like. Strain coupling is also a common mechanism for the coupling between multiple order parameters in a single system. As well as changes on the crystal structure scale, phase transitions modify the microstructure of materials, leading to anomalous mesoscopic features at domain boundaries. The mesostructure of a domain wall is studied experimentally using X-ray diffraction, and interpreted theoretically using Ginzburg-Landau theory. One important consequence of twin mesostructures is their modified transport properties relative to the bulk. Domain wall motion also provides a mechanism for superelastic behaviour in ferroelastics. At surfaces, the relaxations that occur can be described in terms of order parameters and Landau theory. This leads to an exponential profile of surface relaxations. This in turn leads to an exponential interaction energy between surfaces, which can, if large enough, destabilize symmetrical morphologies in favour of a platelet morphology. Surface relaxations may also affect the behaviour of twin walls as they intersect surfaces, since the surface relaxation may lead to an incompatibility of the two domains at the surface, generating large strains at the relaxation. Landau theory may also be extended to describe the kinetics of phase transitions. Time-dependent Landau theory may be used to describe the kinetics of order-disorder phase transitions in which the order parameter is homogeneous. However, the time-dependent Landau theory equations also have microstructural solutions, explaining the formation of microstructures such as tweed.

\section{Introduction}

In crystalline materials, the ideal crystal structure (a motif convolved with an effectively infinite lattice) is often modified by microstructural features such as grain boundaries, twins and dislocations. Here, the term 'microstructure' reflects the fact that these features are generally easily observed with optical or electron microscopy. An important subset of microstructural effects is the consequences of phase transitions and the resulting reduction in crystallographic symmetry. Examples of this are the formation of antiphase domains, due to loss of translational symmetry, and twin domains, due to the loss of point-group symmetry.

Phase transitions therefore have the potential to affect the structure (and, hence, properties) of crystals on three main levels. The most obvious - and extensively studied - is the modification of the crystal structure itself. Then, in order to accommodate the crystal structure change, materials tend to develop microstructures. The third aspect to consider is the local structure in the vicinity of the domain boundaries. The atomic structure at the domain boundary will not, in general, be the same as in the bulk, due to differences in the forces acting on atoms. We thus have an intermediate structural length scale - the mesostructure, as reviewed by Imry (2001). Relevant questions about the mesostructure include (i) what is the local structure of the domain wall, and (ii) over what distance does the domain wall structure relax back to the bulk structure? In a similar vein, atomic arrangements near surfaces differ from the bulk crystal structures. We will argue later that such surface effects are also subtly modified by interactions with phase transitions. 
In this review, we describe progress made in the use of Landau theory to study phase transitions, at both the crystal structure and mesostructure levels. We begin in $\$ 2$ by outlining the derivation of the classical Landau potential from microscopic interactions, and considering the interaction between the order parameter of a phase transition and the associated spontaneous strain. The analysis of microstructures and mesostructures in the context of Landau theory is considered in $\S 3$. We then describe two problems where the properties of domain walls are important: the possibility that the chemical properties of the domain walls will differ, causing different transport properties and allowing chemical reactions to be confined to specific narrow planes; and the interaction between domain wall mobility and macroscopic anelastic behaviour. Relaxed structures at crystal surfaces have obvious parallels with domain boundaries, and in $\$ 4$ we discuss the relaxation of surface structures and the interaction between crystal surfaces and domain walls. In $\S 5$, we examine the relationship between these equilibrium descriptions of phase transitions and microstructures and kinetic descriptions of the non-equilibrium behaviour. We conclude by outlining some key unanswered questions and suggesting some further fields where application of these concepts may prove fruitful.

\section{Theoretical basis}

\subsection{From a microscopic to a macroscopic model}

Although the original applications of Landau-type theories to describe the thermodynamics of phase transitions (Landau, 1937; Devonshire, 1949) were essentially phenomenological attempts to describe the excess free energy as a function of an order parameter $Q$ with mathematically simple functions, the success of this approach to describe phase transitions in real systems has inspired theoretical projects relating macroscopic free-energy functions to fundamental microscopic theory (Chihara et al., 1973; Bruce, 1980; Rae, 1982; Salje et al., 1991; Dove et al., 1992; Radescu et al., 1995; Pérez-Mato \& Salje, 2000). Here, we outline the rather general approach adopted by Salje et al. (1991) and adapted by Pérez-Mato \& Salje (2000).

The starting point is to consider the Hamiltonian of the $\phi^{4}$ model,

$$
\begin{aligned}
H & =\sum_{l} \frac{1}{2 m} p_{l}^{2}+V\left(Q_{l}\right)+\frac{1}{4} \sum_{l, l^{\prime}} v_{l, l^{\prime}}\left(Q_{l}-Q_{l^{\prime}}\right)^{2} \\
V\left(Q_{l}\right) & =E_{0}\left(-2 Q_{l}^{2}+Q_{l}^{4}\right),
\end{aligned}
$$

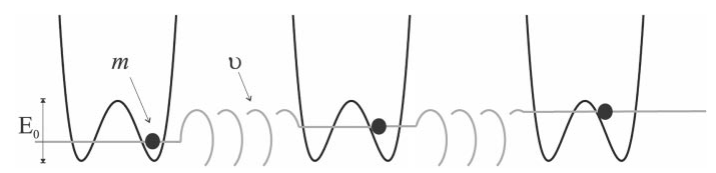

Figure 1

A mechanical analogue to the Hamiltonian for a generic phase transition. The positions of the particles represent the local variables (which become the order parameter in the thermodynamic limit). These particles move in a local potential, and are linked by harmonic springs. where $Q_{1}$ are the local variables (such as the local displacements), $p_{1}$ are the associated momenta and $V\left(Q_{1}\right)$ is the local potential. In a mechanical analogue, as shown in Fig. 1, the three terms in the Hamiltonian represent the kinetic energy of the particles, the potential energy of the surrounding field, and the pair interactions between particles. It is convenient to write the summation of the pair interactions as $v_{l}=\sum_{l^{\prime}} v_{l, l^{\prime}}$.

One important aspect of the Hamiltonian in equation (1) is the balance of $v$ (describing the interparticle interactions) and $E_{0}$ (the local potential). The limits $v \gg E_{0}$ and $v \ll E_{0}$ correspond to extreme displacive and order-disorder phase transitions, respectively. The thermodynamic limit of the model is then found by the Einstein quasi-harmonic approximation. We thus have a characteristic Einstein frequency $\Omega$ and a variance in the order parameter $\sigma$;

$$
\begin{aligned}
\sigma & =\left\langle\left(Q_{l}-\left\langle Q_{l}\right\rangle\right)^{2}\right\rangle=\left(\frac{\hbar}{2 m \Omega}\right) \operatorname{coth}\left(\frac{\hbar \Omega}{2 k_{\mathrm{B}} T}\right) \\
m \Omega^{2} & =v-4 E_{0}+\frac{1}{2} E_{0}\left(Q^{2}+\sigma\right) .
\end{aligned}
$$

The Einstein frequency in the absence of any perturbation is given by $m \Omega_{0}^{2}=v-4 E_{0}$.

In a mean-field model, the Gibbs free energy is related to the Hamiltonian by

$$
G=\operatorname{Tr}\left(\rho H+k_{\mathrm{B}} T \rho \ln \rho\right),
$$

where $\rho$ is the density matrix of states. Using the appropriate Boltzmann expression for $\rho$, and evaluating the terms in the thermodynamic limit, we obtain

$$
\begin{aligned}
G= & N\left\{2 E_{0}(3 \sigma-1) Q^{2}+E_{0} Q^{4}+\frac{1}{2}\left(v-4 E_{0}\right) \sigma+3 E_{0} \sigma^{2}\right. \\
& \left.-\frac{1}{4} \hbar \Omega \operatorname{coth}\left(\frac{\hbar \Omega}{2 k_{\mathrm{B}} T}\right)+k_{\mathrm{B}} T \ln \left[2 \sinh \left(\frac{\hbar \Omega}{2 k_{\mathrm{B}} T}\right)\right]\right\},
\end{aligned}
$$

where $N$ is the number of particles in the system. Although equation (4) does not have a simple general analytical solution, the general case is not difficult to solve numerically. Further progress may be made by considering the distance of a given transition from the displacive limit, $\Delta$ :

$$
\Delta=v / m \Omega_{0}^{2}-1,
$$

so that the displacive limit is $\Delta \rightarrow 0$. Then,

$$
G=6\left[\sigma(T)-\sigma_{C}\right] E_{0} Q^{2}+E_{0} Q^{4}+\ldots
$$

and the mean-square fluctuations are given by

$$
\sigma(T)=\left(\frac{\hbar}{2 m \Omega_{0}}\right) \operatorname{coth}\left(\frac{\hbar \Omega_{0}}{2 k_{\mathrm{B}} T}\right)=\left(\frac{k_{\mathrm{B}} \theta_{S}}{m \Omega_{0}^{2}}\right) \operatorname{coth}\left(\frac{\theta_{S}}{T}\right),
$$

with $\theta_{\mathrm{S}}=\left(\eta \Omega_{0} / k_{\mathrm{B}}\right)$. The Gibbs free energy then becomes

$$
G=\frac{A \theta_{S}}{2}\left[\operatorname{coth}\left(\frac{\theta_{S}}{T}\right)-\operatorname{coth}\left(\frac{\theta_{S}}{T_{C}}\right)\right] Q^{2}+\frac{B}{4} Q^{4}+\ldots,
$$

or, when $T \gg \theta_{S}$,

$$
G=\frac{A}{2}\left(T-T_{C}\right) Q^{2}+\frac{B}{4} Q^{4}+\ldots,
$$

which is the standard classical Landau potential. Equation (8) is thus a Landau-like model that describes the behaviour of 
phase transitions including quantum-mechanical effects at low temperatures.

Having established a microscopic basis for the macroscopic Landau potential, we may go on to consider a number of problems of phase-transition behaviour from a macroscopic point of view. To what extent do the parameters of the Landau free-energy expression have a macroscopic basis? In systems where more than one structural instability is possible, how do they interact? How do the structural changes associated with phase transitions interact with other solid-state phenomena, such as solute atoms or grain surfaces? The underlying simplicity of the Landau potential causes it to be an excellent building block for models of more complex behaviour. One of the most important examples of this is the coupling of order parameters with spontaneous strain effects.

\subsection{Phase transitions and spontaneous strain}

A true proper ferroelastic material is one where the phase transition is strictly associated with the softening of an acoustic phonon, and the order parameter of the transition is the spontaneous strain. Whilst such transitions are rather rare, most solid-state phase transitions have associated changes in the lattice parameters (Carpenter, 2000). As a result, the macroscopic Landau free energy may be decomposed into contributions from the bare order parameter $Q$, the elastic energy of the spontaneous strain tensor $e_{i}$ and the coupling between $Q$ and $e_{i}$ :

$$
\begin{aligned}
G= & \frac{a}{2}\left(T-T_{C}\right) Q^{2}+\frac{b}{4} Q^{4}+\ldots+\sum_{i}\left(\lambda_{1 i} e_{i} Q+\lambda_{2 i} e_{i} Q^{2}\right) \\
& +\frac{1}{2} \sum_{i, k} C_{i k}^{0} e_{i} e_{k} .
\end{aligned}
$$

The values of the coupling constants $\lambda$ and, in particular, which $\lambda$ are permitted to have non-zero values, are constrained by the symmetry of the order parameter. The group-theoretical details of these constraints are beyond the scope of this article, but are reviewed by Carpenter et al. (1998) and Stokes \& Hatch (1988).

Several important points follow from equation (10). The first is that, even in phase transitions that are not proper ferroelastics, a substantial part of the driving force for the phase transition is associated with the spontaneous strain, due to the coupling between $Q$ and $e$. By definition, such interactions have a long correlation length. In the context of the microscopic approach adopted in $\$ 2.1$, the effect of this is to emphasize the long-range correlations within the material and to force phase transitions closer to the displacive limit. As a result, phase transitions whose underlying mechanisms are clearly of an order-disorder type may have macroscopic behaviour which is Landau-like, and hence apparently displacive. Examples of this behaviour include cation ordering in minerals, such as (Al, Si) ordering in feldspars (Salje et al., 1985; Carpenter, 1992; Salje et al., 1993), (Fe, Mg, Mn) ordering in olivine (Redfern, 1998; Redfern et al., 2000), (Al, $\mathrm{Fe}$ ) and $(\mathrm{Ca}, \mathrm{Na})$ ordering in omphacite (Carpenter et al., 1990a). Similarly, the behaviours of many ferroelectric and antiferroelectric systems, such as $\mathrm{BaTiO}_{3}$ (Zalar et al., 2003), squaric acid (Dalal et al., 1998) and KDP (Bussmann-Holder et $a l ., 2001)$ have been observed to depend on a combination of order-disorder and displacive aspects, usually associated with the orientational ordering of a dipole and some sort of surrounding matrix, respectively. Goto \& Lüthi (2003) show that charge-ordering processes in compounds such as $\mathrm{Yb}_{4} \mathrm{As}_{3}$, $\mathrm{Fe}_{3} \mathrm{O}_{4}, \mathrm{NaV}_{2} \mathrm{O}_{5}$ and the $\mathrm{CMR}$ perovskites $\mathrm{La}_{1-x} \mathrm{Sr}_{x} \mathrm{MnO}_{3}$ and $\mathrm{Pr}_{1-x} \mathrm{Ca}_{x} \mathrm{MnO}_{3}$ interact strongly with elastic behaviour. To emphasize the importance of elastic interactions even in cases where crystals are not ferroelastic (e.g. for symmetry reasons), Salje (1990) described such crystals as 'co-elastic'.

The second consequence is that measurements of the spontaneous strain provide a quantitative probe of the order parameter, even when the order parameter is not a displacive soft mode. Starting from equation (10), the equilibrium relationship between $Q$ and $e$ is found by setting $\partial G / \partial e=0$. The most common situations are $\lambda_{1} \neq 0$ and $\lambda_{2}$ is negligible, and $\lambda_{1}=0$ and $\lambda_{2}$ is significant. These two cases lead to $e \propto Q$ and $e \propto Q^{2}$, respectively. Typically, the symmetry-breaking strain (if there is one) behaves as $e \propto Q$, and other strains as $e \propto Q^{2}$, though these rules are not universal. Higher-order couplings between $e$ and $Q$ are allowed but seem to be unimportant in most cases. One geologically important exception is the $P 6_{4} 22 \leftrightarrow P 3_{1} 21$ phase transition in quartz (Carpenter et al., 1988), where comparison of the spontaneous strains with the intensity of the second harmonic signal, the relationships $\left(e_{1}-e_{3}\right) \propto Q^{2}$ and $\left(e_{1}+e_{3}\right) \propto k_{2} Q^{2}+k_{4} Q^{4}$ are observed. This more complex behaviour appears to be related to the combination of tilting, shearing and stretching of the $\mathrm{SiO}_{4}$ tetrahedral units in the phase-transition mechanism.

The third point is that consideration of the spontaneous strain in this way can help rationalize the magnitudes of the observed free-energy coefficients. Given that the spontaneous strain is a function of $Q$ and will relax on a phonon timescale, equation (10) may be recast to eliminate the strain contributions and express the free energy as a function of $Q$ and $T$ alone. The effect of this is to modify, or renormalize, the coefficients of the Landau free energy. As an example, consider the case where $\lambda_{1}$ in equation (10) is zero, and $\lambda_{2}$ is non-zero, so that $e_{i} \propto Q^{2}$. The coupling energy and the spontaneous strain energy are then proportional to $Q^{4}$ and have the effect of reducing the 'bare' value of $B$ to a lower, 'observed' value. The coupling of the spontaneous strain to the order parameter thus helps to justify the observation that many phase transitions with $e_{i} \propto Q^{2}$, such as $\mathrm{SrTiO}_{3}, \mathrm{KMnF}_{3}$, quartz and calcite, are far from the second-order limit $Q^{2} \propto$ $\left|T-T_{C}\right|$, being either near the tricritical point $(B=0)$, or first order $(B<0)$.

The spontaneous strain is also a common mechanism for coupling between order parameters in systems undergoing more than one phase transition. Following Salje (1990), consider two order parameters, each interacting with the spontaneous strain via bilinear coupling [that is the only significant coupling terms in equation (10) are of the form $\left.\lambda_{i 1} e_{i} Q\right]$; 


$$
\begin{aligned}
G= & \frac{a_{1}}{2}\left(T-T_{C 1}\right) Q_{1}^{2}+\frac{b_{1}}{4} Q_{1}^{4}+\ldots+\lambda_{1} Q_{1} e \\
& +\frac{a_{2}}{2}\left(T-T_{C 2}\right) Q_{2}^{2}+\frac{b_{2}}{4} Q_{2}^{4}+\ldots+\lambda_{2} Q_{2} e+\frac{1}{2} C^{0} e^{2} .
\end{aligned}
$$

By applying the stress-free condition $(\partial G / \partial e=0)$, we obtain $C^{0} e=-\left(\lambda_{1} Q_{1}+\lambda_{2} Q_{2}\right)$, and so

$$
\begin{aligned}
G= & \frac{a_{1}}{2}\left(T-T_{C 1}-\frac{\lambda_{1}^{2}}{C^{0}}\right) Q_{1}^{2}+\frac{b_{1}}{4} Q_{1}^{4}+\ldots \\
& +\frac{a_{2}}{2}\left(T-T_{C 2}-\frac{\lambda_{2}^{2}}{C^{0}}\right) Q_{2}^{2}+\frac{b_{2}}{4} Q_{2}^{4}+\ldots-\frac{\lambda_{1} \lambda_{2}}{C^{0}} Q_{1} Q_{2} .
\end{aligned}
$$

The same method applies to other forms of coupling between the order parameters and the spontaneous strain; see Salje et al. (1985), Salje \& Devarajan (1986) and Salje (1990) for details of the mathematical form of $G(Q)$ and the resulting $\left(Q_{1}, Q_{2}\right)$ versus $T$ topologies.

The derivation of order parameter coupling shows that Landau-like models are very well suited to dealing with the interactions between multiple phase-transition processes. A common application of this concept in minerals is to study the effect of some (generally rather slowly equilibrating) cationordering process on a (phonon time scale) displacive change in a mineral's structural framework. Coupling models have been used to describe the interaction between the displacive mode and ( $\mathrm{Al}, \mathrm{Si}$ ) ordering in albite (Salje et al., 1985; Hayward \& Salje, 1996) and anorthite (Phillips et al., 1997), and between the displacive mode and $(\mathrm{Fe}, \mathrm{Mg})$ ordering in pyroxene
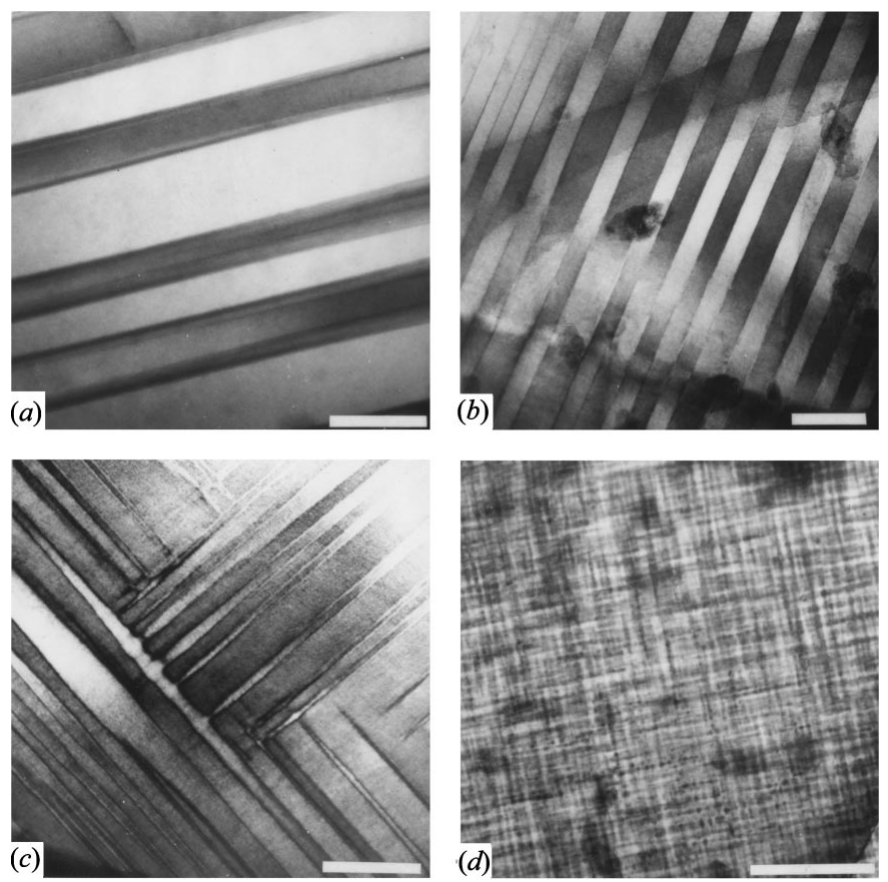

Figure 2

Sequence of domain structures in superconducting $\mathrm{YBa}_{2} \mathrm{Cu}_{3} \mathrm{O}_{7}$ doped with Co. These images show: $(a)$ and $(b)$ simple lamellar twins $(0$ and $1 \%$ Co replacing $\mathrm{Cu}$, respectively); (c) two perpendicular twin orientations with junctions and needles $(2.5 \%)$; and $(d)$ tweed microstructure $(2.8 \%)$. Images from Schmahl et al. (1989). The scale bar is $0.1 \mu \mathrm{m}$.
(Cámara et al., 2003). The same concept has also been applied to the interaction between the antiferroelectric and $\mathrm{ZrO}_{6}$ tilting modes in $\mathrm{PbZrO}_{3}$ (Fujishita et al., 2003), to the two tilt modes (the $M_{3}$ and $R_{25}$ soft modes) in $\mathrm{NaMgF}_{3}$ perovskite (Zhao et al., 1993), and to the interaction between ferroelectricity and $\mathrm{Li}$-ion conduction in $\mathrm{LiNbO}_{3}$ (Lehnert et al., 1997).

Strain interactions also play an important role in controlling the behaviour of materials where the primary interest is in the ferroelectric or ferromagnetic properties of the system. From a microscopic point of view, spontaneous strain interacts with ferroelectric or ferromagnetic behaviour via electron-lattice coupling, as reviewed by Millis (1998). Furthermore, mechanical stresses and strains lead to rather complex microstructures, as described in $\S 3$ below. Bishop et al. (2003) and Ahn et al. (2004) show that the coupling between electronic properties and mechanical stress and strain lead to nanometre-scale textures in the ferroelectric and ferromagnetic domain structures of materials. In systems such as manganite perovskites, the strain textures lead to useful microstructures of metallic and insulating regions.

The term 'multiferroic' was adopted by Schmid (1994) to describe systems that are simultaneously ferromagnetic and ferroelectric. Examples of multiferroic materials include the perovskites $\mathrm{YMnO}_{3}$ and $\mathrm{BiMnO}_{3}$ (Hill \& Filippeti, 2002) $\mathrm{TbMn}_{2} \mathrm{O}_{5}$ (Hur et al., 2004). Such materials have several attractive device applications, resulting from the potential to switch a magnetic state with an electrical field, or vice versa. In particular, multiple state memory elements, exploiting both the magnetic and electrical states of the system, have been envisaged (Wood \& Austin, 1975). However, multiferroics are rare and, from a chemical point of view, it is not obvious that such materials should exist at all. Hill \& Filippeti (2002) note that magnetism requires transition-metal $d$ electrons, which, in a simple model, would destabilize the atomic displacements associated with ferroelectricity. Zheng et al. (2004) have used macroscopic thermodynamic arguments to show that the ferroelectric-magnetic coupling in $\mathrm{BaTiO}_{3}-\mathrm{CoFe}_{2} \mathrm{O}_{4}$ nanostructures has an essentially elastic basis.

\section{Phase transitions, microstructures and mesostructures}

\subsection{Theoretical basis}

Minerals that undergo phase transformations are likely to show microstructures with twin structures as a typical fingerprint for ferroelastic behaviour (Salje, 1990, 2000; Bismayer et al., 1994, 2000; Bosbach et al., 1997; Roewer et al., 1997; Salje et al., 2000). The interaction between twin walls leads to the formation of corner domains, needle domains and comb patterns. These possibilities are illustrated in Fig. 2. Such complex patterns occur on a length scale that bridges the atomic scale and the truly macroscopic scale, a fact that leads to the term 'mesoscopic structures' as being in between these two classic regimes. The patterns are also hierarchical because of the general principle that any pattern can be decomposed into simpler patterns until the most elementary unit, namely 
the twin wall, is found. Twin domains and their boundaries are the dominant microstructures in many materials and have been shown to be important, theoretically and experimentally, for the interpretation of the thermodynamic behaviour of minerals as well as for the design and growth of single crystals and thin films for specific applications.

The application of twin walls to control desirable behaviour in materials or to understand natural behaviour of minerals depends on a proper understanding of the fundamental unit of these hierarchical mesostructures, the twin wall. In the context of the derivation of Landau theory presented in $\$ 2.1$ above, the energy associated with spatial variations of $Q$ is in the $v_{\text {III }}\left(Q_{1}-Q_{I^{\prime}}\right)^{2}$ term. Following a similar argument to the one presented above, we obtain

$$
\begin{aligned}
G= & \frac{A \theta_{S}}{2}\left[\operatorname{coth}\left(\frac{\theta_{S}}{T}\right)-\operatorname{coth}\left(\frac{\theta_{S}}{T_{C}}\right)\right] Q^{2}+\frac{B}{4} Q^{4}+\ldots \\
& +\frac{1}{2} g(\nabla Q)^{2} .
\end{aligned}
$$

The equilibrium variation of $Q(x)$ across a twin wall is then found by Euler-Lagrangian energy minimization. For a limiting second-order phase transition,

$$
\begin{aligned}
Q(x) & =Q_{0} \tanh \left(\frac{x}{w}\right), \\
w & =\left[\frac{2 g}{A \theta_{S}\left(\operatorname{coth}\left(\theta_{S} / T_{C}\right)-\operatorname{coth}\left(\theta_{S} / T\right)\right.}\right]^{1 / 2} .
\end{aligned}
$$

There are slight variations in the solution for tricritical phase transitions, but the tanh profile still holds to a good approximation and the wall width shows essentially the same temperature dependence.

The next step in the exploration of twin wall behaviour is to determine the Ginzburg parameter $g$ or the wall thickness $w$. This task was first attempted using electron microscopy (Yamamoto et al., 1977; Roucau et al., 1979; Boulestieux et al., 1983; Tsai et al., 1992). Electron paramagnetic resonance (EPR) spectroscopy has also been used to probe the structure and thickness of twin walls (Zapart, 2003).

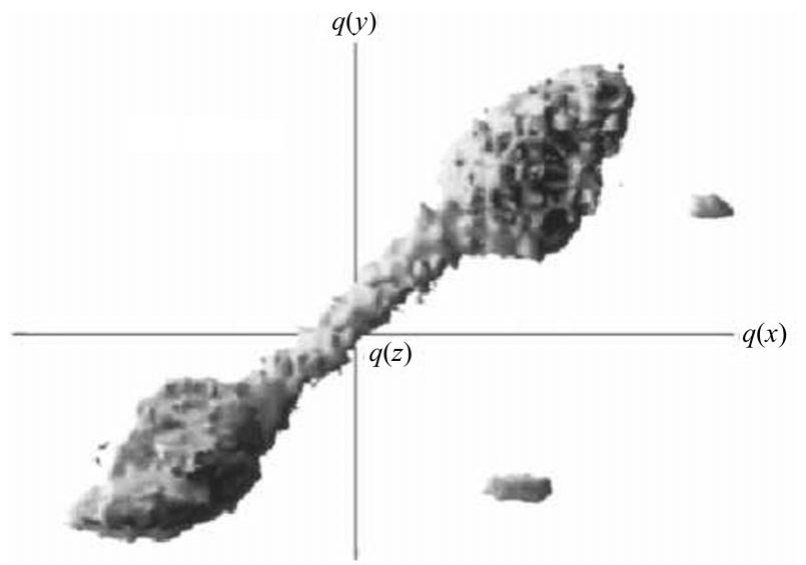

Figure 3

Isointensity surface plot of the $400 / 040$ peak pair in a crystal of $\mathrm{WO}_{3}$ at room temperature after deconvolution with a Gaussian detector response function. The two Bragg maxima are joined by a weak streak of diffraction signal due to the twin wall between the two domains.
Table 1

Values of the twin wall half-width $w$ at room temperature $\left(T \ll T_{C}\right)$ for a number of materials showing ferroelastic domains, measured by X-ray diffraction methods.

\begin{tabular}{lcl}
\hline Material & $w(\AA)$ & Reference \\
\hline $\mathrm{Pb}_{3}\left(\mathrm{PO}_{4}\right)_{2}$ & $<72$ & Wruck et al. $(1994)$ \\
$\mathrm{Pb}_{3}\left(\mathrm{P}_{0.77} \mathrm{As}_{0.23} \mathrm{O}_{4}\right)_{2}$ & 21 & Bismayer et al. $(2000)$ \\
$\mathrm{YBa}_{2} \mathrm{Cu}_{3} \mathrm{O}_{7-d}$ & 7 & Chrosch \& Salje (1994) \\
$(\mathrm{Na}, \mathrm{K}) \mathrm{AlSi}_{3} \mathrm{O}_{8}$ & 13 & Hayward et al. $(1996)$ \\
$\mathrm{WO}_{3}$ & 16 & Locherer et al. $(1998)$ \\
$\mathrm{LaAlO}_{3}$ & 20 & Chrosch \& Salje (1999) \\
\hline
\end{tabular}

X-ray diffraction methods have proved to be a powerful tool to study twinning microstructures and their associated domain boundaries, provided care is taken to optimize the collection and processing of the diffraction signal (Chrosch \& Salje, 1994, 1999; Wruck et al., 1994; Hayward \& Salje, 1996; Locherer et al., 1998). Reciprocal-space studies (that is, diffraction rather than microscopy) are preferable since, although each individual twin boundary occupies a tiny volume (and hence will give a very small experimental signal), diffraction experiments allow for the superposition of the signals from a large number of essentially identical twin walls. The characteristic diffraction pattern from a single twin wall, or a set of parallel twin walls, is a weak streak between two twin related diffraction peaks, as shown in Fig. 3.

Comparing the relative intensities of the bulk and wall diffraction signals allows the twin wall width to be estimated. Results from a number of X-ray experiments are given in Table 1 . These half-widths are typically of the order of $2-5$ unit cells, so that there are up to 10 unit cells within the domain wall. Similar results were obtained in a Monte Carlo simulation study of a [001] $90^{\circ}$ rotation twin wall in $\mathrm{CaTiO}_{3}$ by Calleja et al. (2003), who found $w=5.7 \AA$.

By studying the evolution of the twin wall signal with temperature, the temperature dependence of $w$ has been studied in a disordered ( $\mathrm{Na}, \mathrm{K}$ ) feldspar (Hayward \& Salje, 1996) and the ferroelastic perovskite $\mathrm{LaAlO}_{3}$ (Chrosch \& Salje, 1999). In both these systems, the predicted behaviour $w \propto\left|T-T_{C}\right|^{-1 / 2}$ was observed.

Twin microstructures thus lead to narrow (but not insignificant) volumes that have a different local structure to the bulk crystalline material. Whilst the domain walls are rather extended in two dimensions, they are extremely confined in the third. In the following sections, we explore some of the consequences of these structural effects on the macroscopic properties of twinned materials.

\subsection{Transport and trapping of defects and solutes}

Whereas a structural phase transition reduces the crystallographic symmetry of a material in the bulk, the twin boundaries will act as localized pseudosymmetries, relating one twin domain to another by the lost symmetry operation. It is therefore clear that the local atomic structure of the domain boundary will differ from that of the bulk, and so the chemical and transport properties of the twin walls will also differ from 
those of the bulk. Lee et al. (2003) review the factors that may affect the transport properties of the twin wall. As well as changes in atomic bonding geometry, they note that the density, electric charge and vacancy concentration can vary between the bulk domains and the domain walls. A number of studies have examined these effects experimentally either by observing diffusion in real time or by studying the distribution of elements across a twinned material.

Aird \& Salje (2000) diffused Na into twinned crystals of $\mathrm{WO}_{3}$, to create sheets of the superconducting phase $\mathrm{Na}_{x} \mathrm{WO}_{3}$. In this experiment, $\mathrm{WO}_{3}$ was reduced at $730 \mathrm{~K}$ in the presence of $\mathrm{Na}$ vapour. The different phases were found to have different optical properties, allowing the $\mathrm{Na}$ diffusion to be followed in an optical microscope. The concentration of $\mathrm{Na}$ on the twin walls was confirmed by microprobe analysis, as shown in Fig. 4.

Whilst $\mathrm{Na}$ transport in $\mathrm{WO}_{3}$ is enhanced along the twin boundaries, this effect is not universal. Calleja et al. (2001) simulated the transport of $\mathrm{Na}^{+}$and $\mathrm{Li}^{+}$through a quartz crystal with $\{100\}$ Dauphiné twinning. These results showed that twinning reduced the transport rate along the twin wall (through the [001] channels). This is related to the local structure of quartz at the twin boundary; structural continuity across the twin boundary requires that the [001] channels are somewhat more distorted in the twin wall than in the bulk material. However, transport in the [110] direction was enhanced in the twin walls. On the other hand, Redfern \& Sartbaeva (2002) measured the diffusion of $\mathrm{Li}^{+}$along [001] in quartz, and found that the $\mathrm{Li}^{+}$transport rate had a peak just

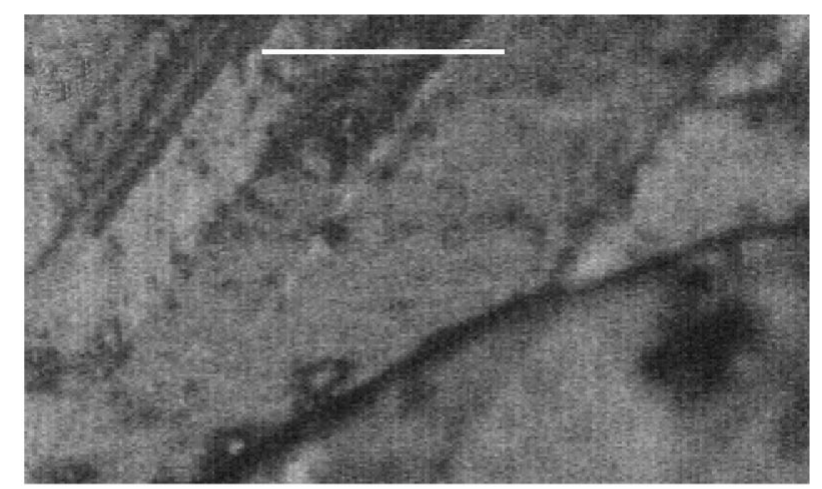

(a)

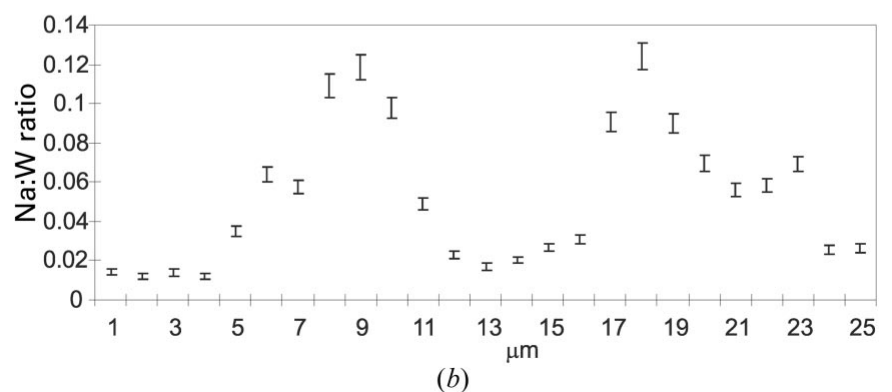

Figure 4

(a) Twinned crystal of $\mathrm{WO}_{3}$, with domains from the tetragonalorthorhombic phase transition. The white line (length $=25 \mu \mathrm{m}$ ) shows the line scanned in the microprobe analysis $(b)$, which shows that the $\mathrm{Na}$ ions are preferentially transported along the twin walls. below the $\alpha-\beta$ phase transition. This peak is evidence that $\mathrm{Li}^{+}$ diffusion is faster along the Dauphiné twin walls than in the bulk material. One possible reason for this discrepancy is that the simulations of Calleja et al. (2001) did not include the possibility that the vacancy concentration may differ between the bulk and wall structures.

Along with the relative diffusion constants along and within the domain walls, there is the question of the relative stability of a given solute species in the bulk phase and in the twin wall. The common observation is that many defects will collect on twin walls, indicating that at least some defect species are stabilized by interaction with the twin wall structure. From this follows the idea of domain wall pinning in ferroelectrics and ferroelastics.

One further consequence of this is the twin memory effect, that a ferroelastic material heated into its paraphase and then cooled back into the ferrophase will often recall its original domain structure. This process is explained by the decoration of the twin wall with defects, which will homogenize relatively slowly in the paraphase structure (Fig. 5).

The first system to have a twin memory effect noted was quartz (Frondel, 1945; Heaney \& Veblen, 1991; Xu \& Heaney, 1997). The Dauphiné twins are pinned strongly by extended defects, such as grain boundaries, dislocations and Brazil twins. There is also a pinning of the Dauphiné twins by point defects. From a kinetic analysis of the loss of this twin memory, these defects were identified as interstitial solute ions within the quartz structure. Voronkova \& Wolf (1993) found that $\mathrm{YBa}_{2} \mathrm{Cu}_{3} \mathrm{O}_{7-x}$ has ferroelastic twin memory, associated with oxygen defects, though it was unclear whether these defects are vacancies or excess $\mathrm{O}$ atoms.

Hayward \& Salje (2000) found twin memory for the albite twins in anorthoclase. Based on the time and temperature dependence of the loss of twin memory in the high-temperature phase, they concluded that the likely atomic mechanism of the twin memory was the collection of $\mathrm{K}$ atoms on the domain boundaries. Cámara et al. (2000) performed a highly sensitive microanalysis of the same mineral sample. These measurements showed an enhancement of $\mathrm{K}$ atoms near the twin walls, and a segregation of $\mathrm{Ca}$ atoms into the bulk. These observations are consistent with the idea that the albite twin

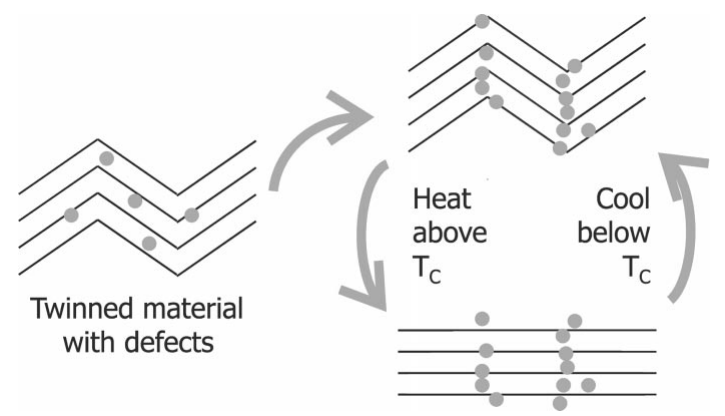

Figure 5

Schematic illustration of atomic scale mechanism of twin memory. Defects that collect on the twin boundary in the ferrophase provide nucleation sites for twin walls when the sample is briefly heated into the paraphase and subsequently cooled. 
wall has a slightly more open crystal structure than the triclinic bulk. However, Parsons et al. (1999) do not find enhanced Ar atom transport along twin walls in feldspar. It is not clear whether this is because Ar atoms are pinned on the walls or that $\mathrm{Ar}$ atoms do not enter the twin walls.

\subsection{Domain walls and anelasticity}

The idea that the motion of domain walls, leading to the growth and shrinkage of domains, is an important mechanism in explaining the macroscopic polarization hysteresis of ferroelectric materials is well established (see, for example, the review of Strukov \& Levanyuk, 1998). One interesting study of this effect is the in situ electron-microscopy study of domains in $\mathrm{BaTiO}_{3}$ under an applied electric field (Snoeck et al., 1994).

The equivalent effect in ferroelasticity has been described as 'superelasticity' (Kityk et al., 2000). For slow strain rates, macroscopic deformation may result from changes in the microstructure of a polydomain material, rather than elastic deformation of atomic bonds. Superelastic materials show hysteresis, substantial softening relative to single-domain elasticity, and strong frequency and temperature dependence, with superelastic softening below the transition temperature and a freezing temperature below which the domains become immobile.

A number of studies of superelasticity exist in the literature, often on systems that are primarily considered as ferroelectric, but which have an associated ferroelastic or co-elastic anomaly. Examples include the softening of $c_{66}$ in $\mathrm{KD}_{2} \mathrm{AsO}_{4}$ (Fally et al., 1995), and internal friction measurements in KDP, DKDP and TGS (Huang et al., 1997). Bourim et al. (2002)

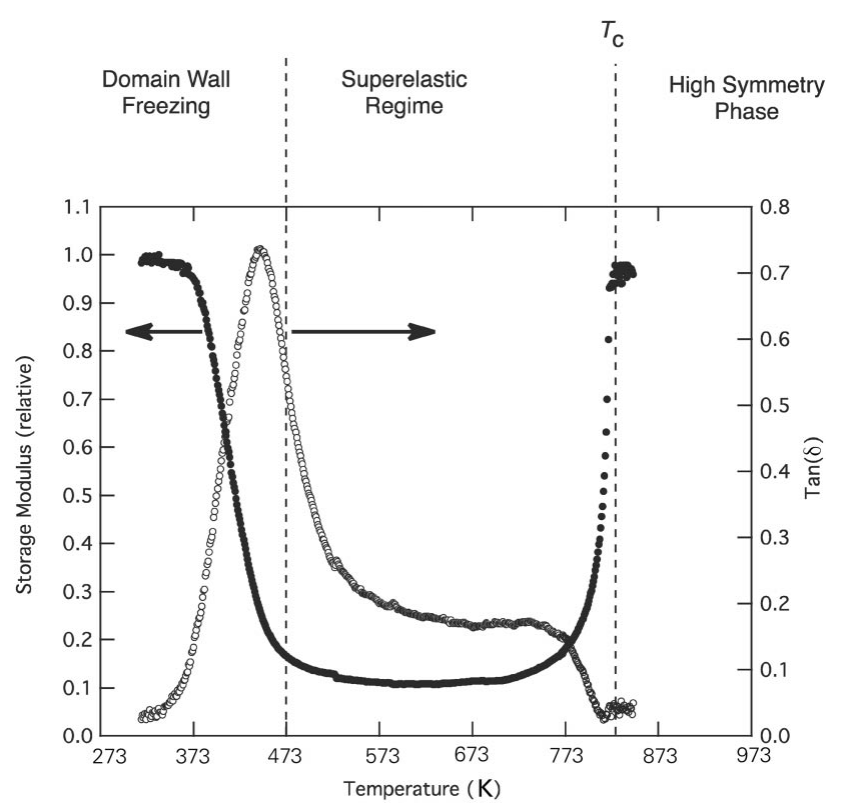

Figure 6

Normalized storage modulus (closed symbols, left axis) and tan $\delta$ (open symbols, right axis) as a function of temperature for $\mathrm{LaAlO}_{3}$. Data from Harrison et al. (2004). have observed anelastic behaviour in PZT, associated with the ferroelectric domains.

An important development of this work was the observation of domain wall softening in $\left(\mathrm{NH}_{4}\right)_{4} \mathrm{LiH}_{3}\left(\mathrm{SO}_{4}\right)_{4}$ (Zimmermann \& Schranz, 1996), which is a pure ferroelastic material. Huang et al. (1997) found evidence of superelasticity in the CuAlZnNi alloy. Kityk et al. (2000) systematically compared the elastic behaviour of $\mathrm{SrTiO}_{3}$ as measured at low frequencies $(10-45 \mathrm{~Hz})$ with the results of ultrasonic and Brillouin scattering experiments, which operate in the $\mathrm{kHz}-$ $\mathrm{GHz}$ range. Schranz et al. (2003) have found superelasticity in $\mathrm{KMnF}_{3}$, though the superelasticity is lost in a sample with $0.3 \% \mathrm{Ca}$ doping. The most likely reason for this is some chemical heterogeneity in the sample, possibly $\mathrm{Ca}$ on the domain walls, leading to strong pinning of the domain walls.

Building on this work, Harrison and co-workers have recently investigated superelastic effects in oxide perovskite systems (Harrison \& Redfern, 2002; Harrison et al., 2003, 2004) as a function of temperature, frequency and composition. Experimentally, the dynamic Young's modulus is represented by a complex number (Schranz, 1997), which may be re-expressed as a storage modulus (the real part of the dynamic Young's modulus) and a loss tangent. Fig. 6 shows the temperature dependence of these quantities in $\mathrm{LaAlO}_{3}$, as measured by Harrison et al. (2004). There is a dramatic softening of $\mathrm{LaAlO}_{3}$ below $823 \mathrm{~K}$, associated with the onset of twinning at the cubic-rhombohedral phase transition (Chrosch \& Salje, 1999). On cooling, $\mathrm{LaAlO}_{3}$ remains superelastic over a wide temperature range. By recording rocking curves of the sample in situ during the dynamic mechanical analysis (DMA) experiments, it has been confirmed that the microstructure of $\mathrm{LaAlO}_{3}$ does evolve dynamically in response to a varying stress, as shown in Fig. 7.

However, at sufficiently low temperatures, a peak in $\tan \delta$ is observed, analogous to a Debye dielectric resonance (Fig. 6). The crossover from superelasticity to frozen domain walls is associated with the pinning of the walls by defects, and is thus affected by the strain rate.

Harrison et al. (2003) found qualitatively similar results for the cubic-tetragonal twin domains in polycrystalline $\mathrm{Ca}_{1-x} \mathrm{Sr}_{x} \mathrm{TiO}_{3}$. In both these systems, the activation energy for domain wall motion (determined from the temperature and frequency dependence of the storage modulus and loss tangent), is of the order of $85-105 \mathrm{~kJ} \mathrm{~mol}^{-1}$, which is comparable with the activation energy for $\mathrm{O}$-atom diffusion through a perovskite structure. O-atom vacancies are therefore the most likely pinning mechanism for ferroelastic domain walls in both $\mathrm{LaAlO}_{3}$ and $\mathrm{Ca}_{1-x} \mathrm{Sr}_{x} \mathrm{TiO}_{3}$ perovskites. Lagraff \& Payne (1992) found that the ferroelastic domain mobility in $\mathrm{YBa}_{2} \mathrm{Cu}_{3} \mathrm{O}_{7-\delta}$ was also constrained by $\mathrm{O}$-atom mobility, and simulations by Calleja et al. (2003) found that $\mathrm{O}$-atom vacancies were stabilized on twin boundaries in $\mathrm{CaTiO}_{3}$, with $\Delta E=1.2 \mathrm{eV}$ between $\mathrm{O}$-atom vacancies in the bulk and in the twin wall. Whilst tetragonal $\mathrm{Ca}_{1-x} \mathrm{Sr}_{x} \mathrm{TiO}_{3}$ shows superelastic behaviour, the orthorhombic phase appears not to be superelastic to a significant degree. The reasons for this are, at present, unclear. 


\section{Surfaces}

Modelling the surface of crystals is important for understanding properties such as morphology, catalytic properties and all transport from outside the crystal. The atomic scale structure of a surface can differ topologically from the unrelaxed surface or not. Topological changes to the surface are called surface reconstruction, non-topological changes are called surface relaxation. In this section, we focus on surface relaxations. Including the effects of surface relaxations in calculations of surface or attachment energies can improve the accuracy of morphological predictions. For a monoatomic solid, it can be shown that, provided that the interaction potentials can be approximated by a second-order Taylor series in the atomic displacements, the atomic displacements are exponential in form, possibly modulated by an oscillatory envelope with a wavelength that need not be commensurate with the lattice. Including the effect of chemical degrees of freedom increases the length scale of the relaxation and may

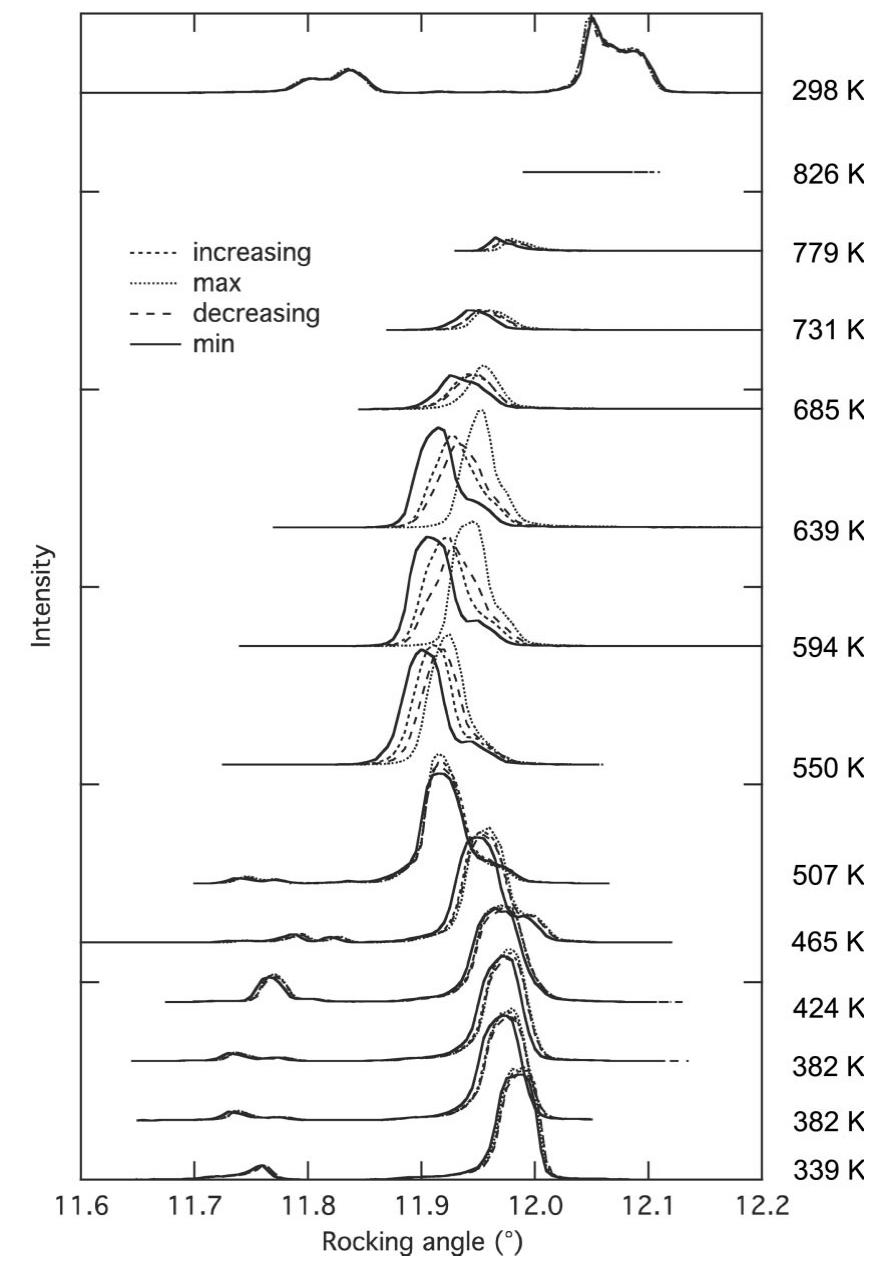

Figure 7

Stroboscopic XRD rocking curves as a function of temperature for $\mathrm{LaAlO}_{3}$, recorded at $1 \mathrm{~Hz}$. Each peak in the rocking curve represents a different ferroelastic domain orientation. The four components of the rocking curve correspond to different parts of the dynamic stress cycle: solid line: minimum force $\sim 20 \mathrm{mN}$; dotted line: maximum force $\sim 380 \mathrm{mN}$; medium-dashed line: maximum rate of increasing force; long-dashed line: maximum rate of decreasing force. From Harrison et al. (2004). increase or decrease the magnitude of the displacements at the surface. The overlap of surface relaxations from different surfaces can cause the surfaces to interact, the interaction energy also being exponential in nature. If the interaction energy is sufficiently large compared to the surface energy then, for small crystal sizes, the symmetrical growth morphology is destabilized and a platelet morphology is stabilized. It is not known however whether this effect occurs in practice. Surface relaxations also affect the properties of microstructural units such as twin walls and needle domains that intersect the surface.

At a surface, there are two types of phase-transition-related phenomena that can occur. The first is reconstruction, a topological change of the surface. The second is relaxation, a distortion of the surface that does not result in any topological changes. Fig. 8 illustrates generic examples of surface relaxations and reconstructions. In this section, we focus on the second of these phenomena: the surface relaxation. For more information about surface reconstruction, the reader is referred to Zangwill (1988), LaFemina (1992) and Lüth (1993). The surface relaxation results in a lowering of the energy of a surface from that of a truncated bulk crystal. Attempting to measure the atomic scale details of the surface relaxation by experiment has the same difficulties as measuring the structure of domain walls (see $\$ 3$ above). Although surface relaxations can sometimes be detected by the broadening of diffracted peaks (e.g. Harrison \& Salje, 1994), the atomic scale details of surface relaxations are hard to measure directly by experiment, but can be investigated by simulation studies.

Simulations of surfaces make use of either $a b$ initio or interatomic forcefield calculations. These models can be used to predict morphologies and how morphologies are affected by additives, as well as investigating the binding energies of surface adsorbed species, see for example Gay \& Rohl (1995), Titiloye et al. (1993), Parker et al. (1993) and de Leeuw \& Parker (1997). Relaxation of the surface is important in performing these calculations since the energy of relaxation is comparable with the differences between the surface and attachment energies of candidate surfaces, leading to different morphologies being calculated for relaxed and unrelaxed surfaces. There are two recipes for calculating growth morphologies (see for example Gay \& Rohl, 1995), both of which make use of the Wulff plot (Wulff, 1901). Equilibrium

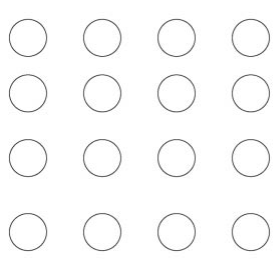

(a)

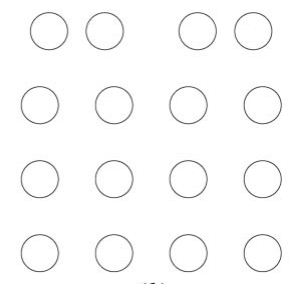

(b)
Figure 8

Generic examples of a surface relaxation and a surface reconstruction (Philips 2001; Lüth, 1993). 
morphologies are calculated from surface energies and are considered to apply to crystal nuclei of sizes smaller than about a micrometre. Growth morphologies are calculated from attachment energies (the energy needed to add an extra layer of unit cells to a crystal) and are considered applicable to larger crystals (Hartman \& Bennema, 1980).

Mackrodt et al. (1987) calculated equilibrium morphologies from unrelaxed and relaxed surfaces for $\alpha-\mathrm{Al}_{2} \mathrm{O}_{3}$ and $\alpha-\mathrm{Fe}_{2} \mathrm{O}_{3}$. They found substantial differences between the relaxed and unrelaxed morphologies, highlighting the importance of including surface relaxations in morphological calculations. Gay \& Rohl (1995) calculated growth morphologies of the minerals zircon, quartz and corundum. They used the program MARVIN (Gay \& Rohl, 1995) to carry out calculations of the attachment energies of relaxed surfaces. Their calculated growth morphologies of zircon and quartz were consistent with those found in natural samples. Their corundum morphology was not the same as that observed in natural corundums. The explanation for this discrepancy is believed to be due to the adsorption of foreign atoms onto $\{111\}$ surfaces during growth (Hartman, 1989).

One question one can ask oneself is, what generic form do surface relaxations take? Are surface relaxations in different crystals expected to have any features in common? Starting from a general model of interacting layers of atoms parallel to a free surface, Houchmandzadeh et al. (1992) determined a universal exponential profile for the surface relaxation. The only assumption that goes into the proof is that the interactions between the planes of atoms can be expanded as a Taylor series. The simplest non-trivial model of this class is shown in Fig. 9. In this model, there are interactions between firstneighbour and second-neighbour planes: these interactions are necessary and sufficient to produce a surface relaxation of finite width. (The model that only has nearest-neighbour interactions is indifferent to the presence of a surface.) Nearest neighbours are connected by springs described by a spring constant of $k$ and an equilibrium length of $a$, second neighbours are connected by springs of spring constant $K$ and equilibrium length $A$. The spring parameters may be interpreted as the result of a second-order Taylor expansion of the energy of interaction between the planes. For a surface relaxation to occur, $2 a \neq A$.

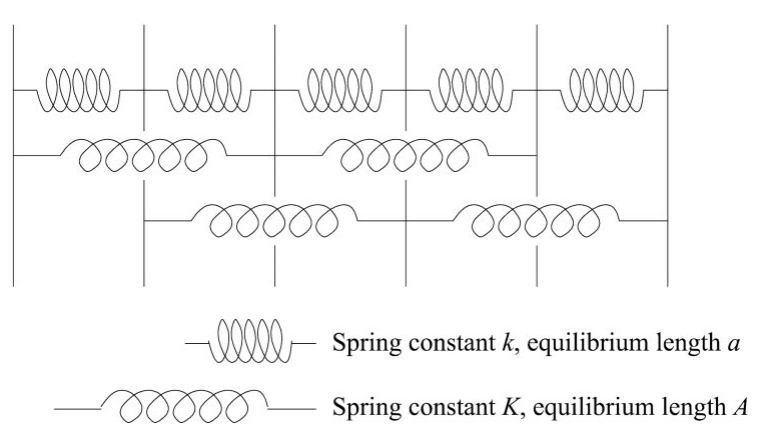

Figure 9

The simplest model with a long-range surface relaxation. It consists of atomic layers parallel to the surface. Nearest-neighbour and secondneighbour layers interact harmonically.
The surface relaxation takes the form

$$
\varepsilon=\varepsilon_{0} f_{n} \exp (-n / \lambda),
$$

where $n$ is the layer number (starting with 0 at the surface), the modulating function $f_{n}$ is $( \pm 1)^{n}$. Which form is taken depends on the relative values of $k$ and $K$. The phase diagram of this system is shown in Fig. 10. Examples of relaxation profiles are shown in Fig. 11. This model may also be used to investigate phase transitions in the system. If the spring constant $K>0$ and the constant $k$ is decreased, the length scale of the oscillatory relaxation, $\lambda$, increases, while the magnitude of the relaxation $\varepsilon_{0}$ decreases. At the point $k=0$, the length scale becomes infinite and the magnitude becomes zero. Beyond this point, higher-order interactions stabilize an antiferroelastic phase. A similar behaviour takes place in the $K<0$ part of the phase diagram as the $K=-k / 4$ line is approached, except that the new phase produced on crossing the line is ferroelastic.

Two generalizations to the above model are possible. The first is that interactions between third or more distant neighbour planes may be significant. The second is that the substance need not be monoatomic. If relaxations between more distant neighbour planes are significant then the modulating factor $f_{n}$ takes the form of a sinusoidal modulation. This modulation need not be commensurate with the lattice, in which case the phase transition that occurs on crossing a stability line on the phase diagram may produce an incommensurate phase. If the solid is not monoatomic then the surface relaxation has more degrees of freedom, e.g. the surface relaxation can involve polarization as well as strains. However, the displacements of the atoms will still be given by linear combinations of modulated exponentials.

The picture of surface relaxations may be generalized by an appeal to the Landau theory of phase transitions, see $\$ 2$ (Lee et al., 1999, 2000). Phase transitions and surfaces share the common feature of a broken symmetry. In the case of a surface, the symmetry is broken at the surface and restored in the bulk of the material. This broken symmetry can be measured by one or more symmetry-adapted order parameters. A Landau-Ginzburg free energy can be used to describe the behaviour of the order parameter(s) measuring this

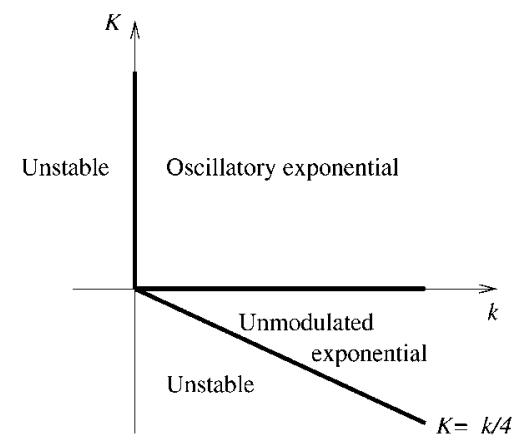

Figure 10

Phase diagram of the system shown in Fig. 9. The possible stable states of the system are $(a)$ an oscillatory exponential relaxation or $(b)$ an unmodulated exponential relaxation. 
symmetry breaking. In the case in which a single order parameter is needed, the Landau-Ginzburg free energy takes the form

$$
G[Q]=-\alpha Q(0)+\int_{0}^{\infty}\left[\frac{a}{2} Q^{2}+\frac{g}{2}\left(\frac{\partial Q}{\partial z}\right)^{2}\right] \mathrm{d} z .
$$

The first term describes the surface: the broken symmetry at the surface allows terms linear in the order parameter. The integral is the usual Landau-Ginzburg form, the coefficient $a$ is positive indicating that minimum free energy of the system is that in which the symmetry broken at the surface is restored. Because $a$ is positive, terms of higher order in $Q$ are not needed. In general, more than one symmetry will be broken by the surface, in which case several order parameters are needed.

The Landau-Ginzburg free-energy description of the surface relaxation readily allows chemical degrees of freedom to be included. If some chemical process (e.g. incorporation of substitutional atoms) has the same symmetry as the surface relaxation order parameter, there will be linear coupling terms between the two (Lee \& Salje, 2000). The free energy becomes

$$
\begin{aligned}
F[Q, \xi]= & -\alpha Q(0)-\beta \xi(0) \\
& +\int_{0}^{\infty}\left[\frac{a}{2} Q^{2}+\frac{b}{2} \xi^{2}+c Q \xi+\frac{1}{2}\left(\frac{\partial Q}{\partial z}\right)\right] \mathrm{d} z,
\end{aligned}
$$

where $\xi$ is a chemical order parameter. The coupling between the order parameter and the concentration will result in an increase in the length scale of the relaxation. The magnitude of the relaxation at the surface will be either increased or decreased depending on the coefficient $\beta$.

A consequence of the finite size of the surface relaxation is that, in crystals of size comparable to the length scale of the surface relaxation, there will be an interaction energy between relaxations at opposite surfaces (Lee et al., 1999). The interaction energy will take the form of an exponential, modulated by $f_{n}$ and may be attractive or repulsive depending on the symmetry of the relaxation. For example, a strain relaxation of even symmetry will give an attractive interaction, whereas a polarization relaxation of odd symmetry will give a repulsive

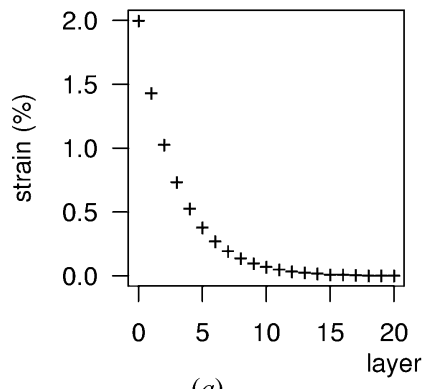

(a)

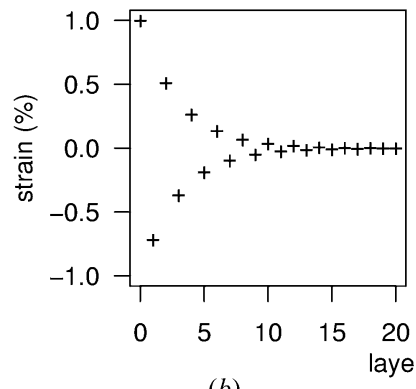

(b)
Figure 11

Modulated and unmodulated exponential relaxations. In the model shown in Fig. 9, the only possible modulations are (a) ferroelastic or $(b)$ antiferroelastic. In models in which third or higher neighbour interactions are significant, incommensurate modulations may occur. interaction. This interaction, if of sufficient magnitude, may result in the growth of crystals with a platelet morphology, even if that morphology is of lower symmetry than the point group of the crystal.

This can be seen by considering a crystal of cubic symmetry for which the $\{100\}$ surfaces have the lowest surface energy. The free energy of a growing crystal nucleus with edges $a, b$ and $c$ will have the form

$$
\begin{aligned}
G= & 2 \sigma(a b+b c+c a)+2 \eta \sigma[a b \exp (-c / \lambda)+b c \exp (-a / \lambda) \\
& +c a \exp (-b / \lambda)]-\gamma a b c,
\end{aligned}
$$

in which $\sigma$ is the surface energy of a (100) surface in an infinite crystal, $\eta$ measures the size of the interaction energy between surfaces relative to the surface energy and $\gamma$ is the bulk free energy of the crystal. The growth of the crystal can be determined by gradient flow equations of the form

$$
\begin{aligned}
& \frac{\partial a}{\partial t}=-\frac{1}{b c} \frac{\partial G}{\partial a} \\
& \frac{\partial b}{\partial t}=-\frac{1}{c a} \frac{\partial G}{\partial b} \\
& \frac{\partial c}{\partial t}=-\frac{1}{a b} \frac{\partial G}{\partial c} .
\end{aligned}
$$

If the parameter $\eta$ is positive or greater than -1 , the interaction between the surfaces has no effect on morphology. Small crystal nuclei dissolve and larger nuclei grow into macroscopic crystals of cubic morphology. However, if $\eta$ is smaller than -1 , small nuclei grow into platelets while nuclei large compared with the range of the interaction between the surfaces grow into macroscopic cubic crystals as before. These results can be seen in Fig. 12, which shows the gradient flow directions.

This mechanism has been proposed as an explanation of cases where platelet morphologies of lower symmetry than the point group of the crystal have been observed, e.g. growth of hydroxyapatite in bones (Lee et al., 2002) and the crystallization of apoferritin (Yau \& Vekilov, 2000). However, at the

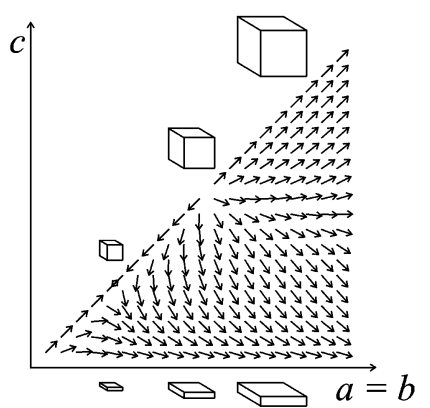

(a)

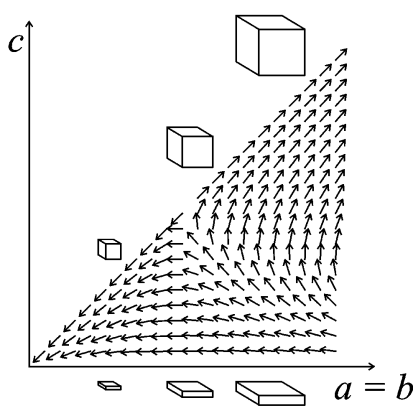

(b)
Figure 12

Growth of cubic and platelet crystals. The edge lengths of the crystals are $a=b$ and $c$. No arrows are shown for needle morphologies $c>a$ because these morphologies are unstable. Arrows show gradient flow directions for two cases. (a) $\eta>-1$, showing the usual case of either growth into macroscopic crystals with morphologies determined by the point group of the crystal, in this case cubes or dissolution. (b) $\eta<-1$. In this case, whereas large nuclei grow into macroscopic cubes as before, small nuclei grow into platelets. 
time of writing this proposal remains speculative. Calculations of the surface relaxation of the (100) surface of hydroxyapatite have been carried out showing that the polarization perpendicular to the surface and the interaction between two opposite surfaces show modulated exponential behaviours (Lee et al., 2000), as shown in Fig. 13.

Microstructural features such as twin walls may intersect with surfaces. Understanding the behaviour of a twin wall intersecting a surface is of great importance for understanding the diffusion of particles from the surface into the bulk if, as discussed in \$3.2, domain walls are the primary conduits of chemical transport into the bulk. The behaviour of twin walls intersecting a surface will be affected by two factors, firstly the surface relaxation (Novak \& Salje, 1998; Conti \& Salje, 2001) and secondly by secondary strains coupling to the order parameter (Lee et al., 2002). So far investigations have been limited to the case of domain walls intersecting a surface at $90^{\circ}$.

The influence of a surface relaxation on the surface structure of a domain wall has been investigated both numerically (Novak \& Salje, 1998) and analytically (Conti \& Salje, 2001). The system under investigation shows a tetragonal to orthorhombic transition by a shear in the tetragonal plane. At the surface, the equilibrium shear is different to the equilibrium shear in the bulk, thus there is a surface relaxation. The key fact in determining the surface relaxation of the wall is that the surface relaxations are not compatible at the surface; see Fig.

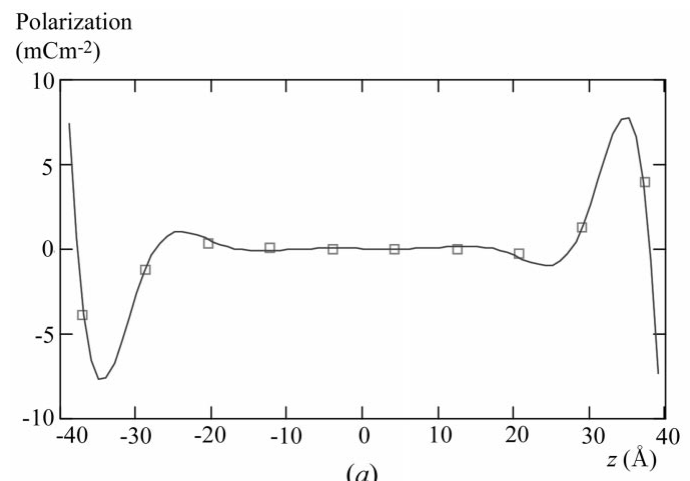

(a)

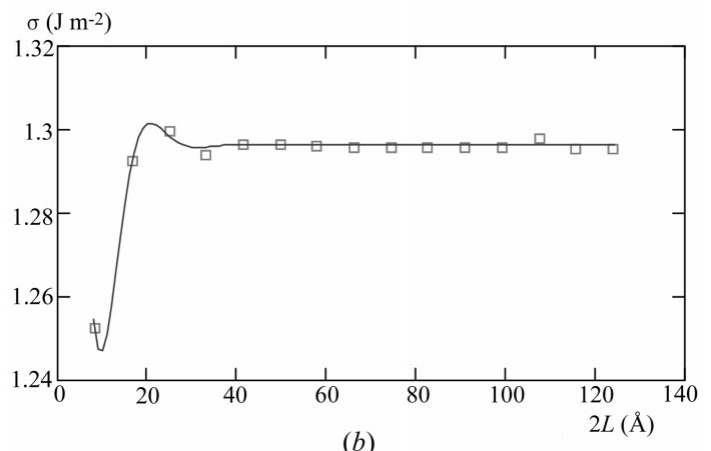

(b)

Figure 13

Surface relaxations in hydroxyapatite with two (100) surfaces. (a) shows the polarization of a system ten layers thick, fitted to an oscillatory exponential relaxation at each surface. $(b)$ shows the interaction energy between surfaces fitted to an oscillatory exponential (Lee et al., 2000).
14. The lines of isostrain reveal that the domain wall has a trumpet-like profile close to the surface, first narrowing and then widening (Fig. 15). So far experimental determination of the twin wall profile by atomic force microscopy has been constrained by the low resolution of the technique (Whittborn et al. 2002).

A second factor that can influence the surface structure of a twin wall is the presence of a secondary strain across a domain wall, e.g. a volume strain $\varepsilon_{11}+\varepsilon_{22}$ which shows a linearquadratic coupling to the shear strain order parameter (Lee et al., 2002). In the bulk, the strain compatibility condition ensures that, while $\varepsilon_{11}$ can vary across the wall, $\varepsilon_{22}$ is constrained to be zero. There is a stress $\sigma_{22}$ associated with this unrelaxed shear. At the surface, a more general relaxation is possible with the boundary condition requiring that $\sigma_{22}=0$. The result of this relaxation is quite different and the strains at the two surfaces are asymmetric, which is not the case for a surface relaxation.

The effect of the surface on other more complex mesoscopic structures such as needle domains has also been investigated. Novak et al. (2002) investigated the elastic behaviour of a needle domain at various depths below the surface. They found that, as the domain wall approached the surface, a region of reduced order parameter extended from the needle tip to the surface, followed by the break-up of the needle into two separate domain walls, see Fig. 16, influencing the surface topography. They were also able to compare their results for needles far from the surface with the quadratic profile predicted by non-local continuum elasticity theory (Salje \& Devarajan, 1986; Pertsev et al., 2000), see Fig. 17.

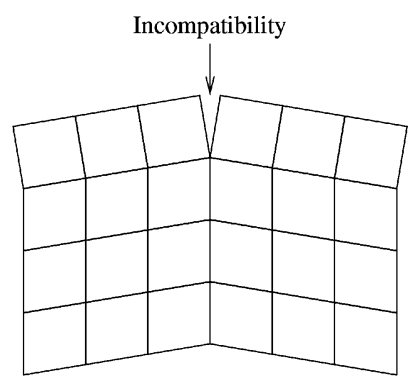

Figure 14

The key factor in determining the surface structure of a domain wall intersecting a surface at $90^{\circ}$ is the incompatibility of the surface relaxations at the twin wall.

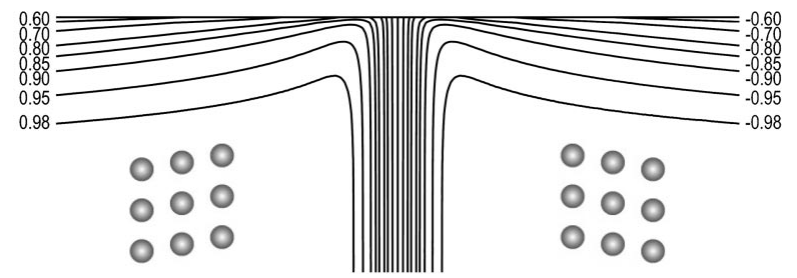

Figure 15

Lines of isostrain at the intersection of a twin wall and a surface (Novak \& Salje, 1998). 


\section{Kinetics}

The equations describing the kinetics of a phase transition can be derived from a master equation describing transitions between configurations of local order parameters $q_{i}$. This equation can be coarse grained to produce equations describing the time evolution of the macroscopic order parameter $Q$. The form of these equations depends on two factors, namely, whether the local order parameters are continuous or discrete, and whether the order parameter is a conserved quantity. If the local order parameter is continuous then the dynamics of the system are described by timedependent Landau theory, which interpolates between complete conservation and non-conservation of the order parameter. In these limiting cases, the Cahn-Hilliard equation and the Ginzburg-Landau equations, respectively, can be recovered as special cases of the time-dependent Landau equation. If the order parameter is discrete then the time evolution of the order parameter is described by IsingKawasaki or Ising-Glauber dynamics, depending on whether the order parameter is conserved (Ising-Kawasaki) or not (Ising-Glauber). Although Ising dynamics and time-dependent Landau theory predict different rates of ordering far from $T_{C}$, it is difficult to obtain experimental data in this region, making it difficult to decide which of the two rate laws is applicable to transitions. Microstructural solutions, e.g. stripes and tweed, result from the kinetics of phase transitions in which the order parameter is conserved or partially conserved.

The starting point for deriving equations describing the kinetics of the phase transitions is a master equation describing transitions between microscopic states of the system. This equation only contains the assumption that the state of the system can be described by a vector of local order

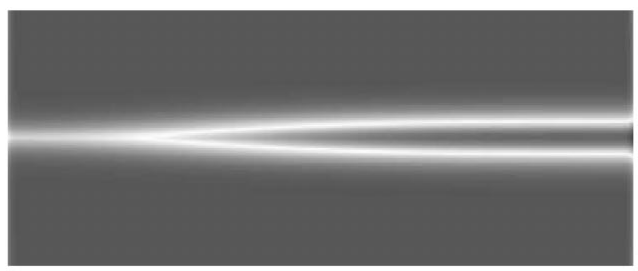

(a)

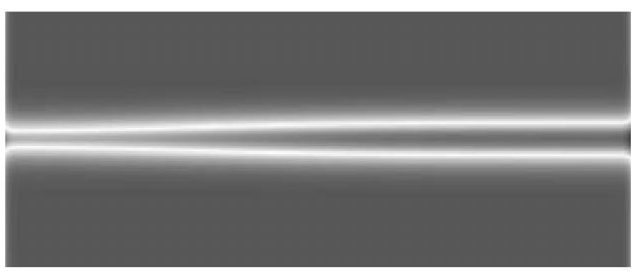

(b)

\section{Figure 16}

Needle domain close to a surface. Dark colours indicate domains, light colours domain walls. The left side of the figure is a free surface. (a) As the needle domain approaches the surface, the needle tip extends to touch the surface. (b) When the needle domain approaches the surface more closely, it breaks up into two separate twin walls (Novak et al., 2002). parameters $\mathbf{q}$ and that the evolution of the system is Markovian, i.e. that the state of the system at time $t+\mathrm{d} t$ depends only on its state at time $t$. Thus the system has no memory. The master equation takes the form (Glauber, 1963)

$$
\frac{\mathrm{d} P(\mathbf{q}, t)}{\mathrm{d} t}=\sum_{\mathbf{q}^{\prime}}\left[\Gamma\left(\mathbf{q}^{\prime} \rightarrow \mathbf{q}\right) P\left(\mathbf{q}^{\prime}, t\right)-\Gamma\left(\mathbf{q} \rightarrow \mathbf{q}^{\prime}\right) P(\mathbf{q}, t)\right],
$$

where $\mathbf{q}$ is a vector describing the state of the system (its components $q_{i}$ may be thought of as a set of local microscopic order parameters). $P(\mathbf{q}, t)$ is the probability that at time $t$ the system is in the state $\mathbf{q}$. The coefficients $\Gamma\left(\mathbf{q}^{\prime} \rightarrow \mathbf{q}\right)$ and $\Gamma\left(\mathbf{q} \rightarrow \mathbf{q}^{\prime}\right)$ describe the probability per unit time that the system makes transitions from the state $\mathbf{q}^{\prime}$ to the state $\mathbf{q}$ and from the state $\mathbf{q}$ to the state $\mathbf{q}^{\prime}$, respectively. To convert this equation into an equation describing the time evolution of the macroscopic order parameter $Q$, we must coarse-grain the equation, integrating out microscopic degrees of freedom. The results of this procedure depend on whether the microscopic order parameters $q_{i}$ are discrete or continuous and whether or not the order parameters are conserved.

\subsection{Ising dynamics}

In the case where only transitions between discreet states of the system are allowed, coarse graining the master equation (20) results in Ising dynamics. There are two important special cases of Ising dynamics. These are Ising-Kawasaki (Kawasaki, 1966) and Ising-Glauber (Glauber, 1963). In Ising-Kawasaki dynamics, the order parameter is conserved and the most important type of transition that the system makes is the exchange of two adjacent spins. In Ising-Glauber dynamics, the order parameter is not conserved and the principal type of transition the system makes is the flipping of a single spin. These two processes are illustrated in Fig. 18. The equations

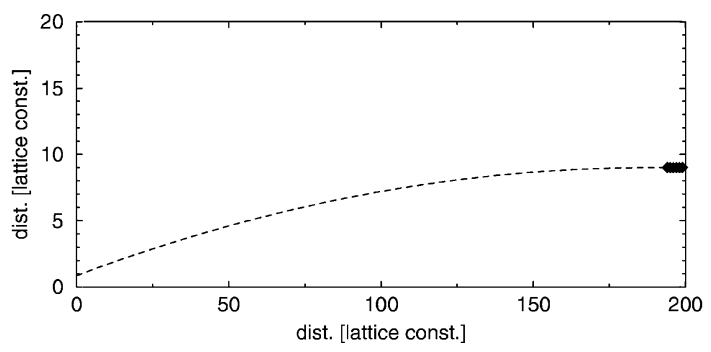

Figure 17

Comparison of the calculated needle wall trajectory with the quadratic profile predicted by theory (Salje \& Ishibashi, 1996; Pertsev et al., 2000).

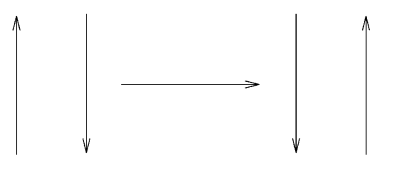

$(a)$

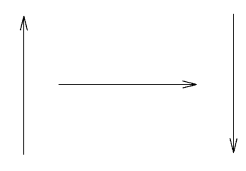

(b)
Figure 18

Transitions in Ising models. (a) In Kawasaki dynamics, the order parameter is conserved and equilibration is achieved by neighbouring sites exchanging spins. (b) In Glauber dynamics, the order parameter is not conserved and equilibration is achieved by individual sites changing their spin states. 
describing the time evolution of the macroscopic order parameter for Kawasaki and Glauber dynamics, respectively, in the mean field limit are

$$
\begin{aligned}
& \frac{\mathrm{d} Q}{\mathrm{~d} t}=-\mu\left[\frac{2 Q}{Q^{2}+1}-\tanh \left(\frac{Q T_{C}}{T}\right)\right] \\
& \frac{\mathrm{d} Q}{\mathrm{~d} t}=-\mu\left[Q-\tanh \left(\frac{Q T_{C}}{T}\right)\right] .
\end{aligned}
$$

\subsection{Time-dependent Landau theory}

In the case in which continuous transitions of the local order parameters are allowed, coarse graining of the master equation (20) introduces two length scales. The first length scale is the correlation length $\xi$ (Meitu et al., 1976a,b). This describes the length scale of interactions in the system. The second length scale is the conservation length $\xi_{C}$ (Salje, 1988; Carpenter \& Salje, 1990), describing the length scale over which the order parameter is conserved. This parameter allows interpolation between the extreme cases of an unconserved order parameter $\xi_{C}=0$ and a fully conserved order parameter $\xi_{C}=\xi$. The time-dependent Landau equation is given by (Marais \& Salje, 1991)

$$
\frac{\mathrm{d} Q}{\mathrm{~d} t}=-\mu\left[1-\frac{\xi_{C}^{2}}{\xi^{2}} \frac{\sinh (\xi \nabla)}{\xi \nabla}\right] \frac{\partial G}{\partial Q},
$$

where $\mu$ is a mobility with Arrhenius behaviour, the activation energy $G^{*}$ may depend on the value of the order parameter. $G$ is a Landau free energy describing the phase transition. The meaning of the differential operator inside the sinh function can be interpreted as meaning the series expansion of the function in terms of the operator. Time-dependent Landau theory gives two important equations used in the study of phase-transition kinetics as special cases. In the fully conserved case $\xi_{C} \rightarrow \xi$, the Cahn-Hilliard equation (Cahn, 1961, 1962, 1968; Hilliard, 1970; Langer, 1971) is recovered when equation (22) is expanded to lowest order in $\xi$ :

$$
\frac{\mathrm{d} Q}{\mathrm{~d} t}=\mu \xi_{C}^{2} \nabla^{2}\left(\frac{\partial G}{\partial Q}\right)
$$

The Ginzburg-Landau equation (Lifshitz \& Pitaevskii, 1981) is obtained in the limit $\xi_{C} / \xi \rightarrow 0$, in which the order parameter is not conserved:

$$
\frac{\mathrm{d} Q}{\mathrm{~d} t}=-\mu \frac{\partial G}{\partial Q} .
$$

One example of the use of the Ginzburg-Landau equation is given by the kinetics of cation ordering in omphacite (Carpenter et al., 1990b). Carpenter et al. calculated order parameters from superlattice reflection intensities and compared them with solutions to the Ginzburg-Landau equation, Fig. 19. The structural changes due to the phase transition required them to use an order parameter dependent on $\mu$.

An important question that has to be addressed is the location of the changeover point between Ising kinetics and time-dependent Landau theory. At what point can an order parameter be treated as 'continuous'? The Ising model itself clearly must be described in terms of a discrete order parameter. However, the generalization of the Ising model, the $n$-state Potts model, in which each spin site can be in one of $n$ states, is well described by continuous order parameter kinetics for $n>4$ (Salje \& Marais, 1992).

Close to the transition temperature, Ising kinetics and timedependent Landau theory predict similar evolution rates of the order parameter; it is only for temperatures $T \sim 0.5 T_{C}$ that the two rate laws show significant degrees of divergence, with time-dependent Landau theory predicting faster kinetics than Ising-Kawasaki and Ising-Glauber. Molecular dynamics simulations show that time-dependent Landau theory correctly predicts the ordering kinetics of a system with a continuous order parameter, while Ising kinetics predictions are too slow (Marais, Heine \& Salje, 1991). Experimental studies of processes such as cation ordering in minerals are typically performed at rather high temperatures (often of the order of $0.9 T_{C}$ ), so that the thermally activated mechanisms proceed at a reasonably fast rate. Unfortunately, the predictions of Ising kinetics and time-dependent Landau theory are practically indistinguishable in this temperature range, so most kinetic experiments are unable to distinguish between these different paradigms of kinetic behaviour. A study was undertaken of $\mathrm{Al}-\mathrm{Si}$ ordering in $\mathrm{CaAl}_{2} \mathrm{Si}_{2} \mathrm{O}_{8}$, with a very high transition temperature $T_{C} \simeq 2800 \mathrm{~K}$ (Salje et al., 1993). This unusually high $T_{C}$ made it possible to study ordering at $0.5 T_{C} \simeq 1400 \mathrm{~K}$. A fit of both equilibrium and kinetic data to Ising-Glauber kinetics and time-dependent Landau theory showed a good match for time-dependent Landau theory (Fig.

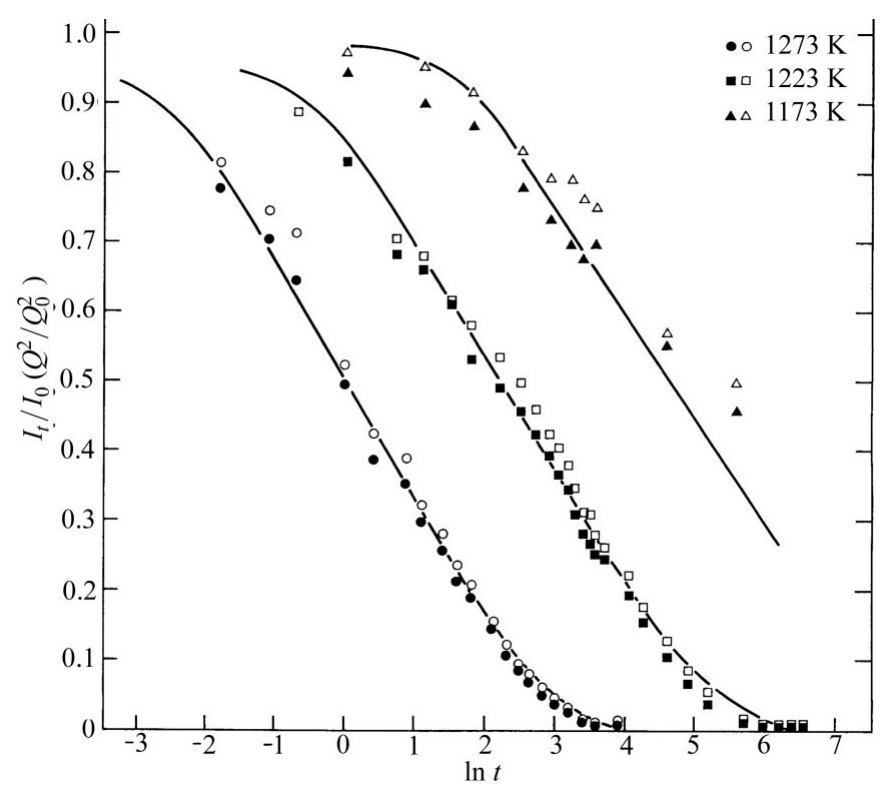

Figure 19

Comparison between experimental cation ordering in omphacite and solutions to the Ginzburg-Landau equation. Reprinted with permission from Carpenter et al. (1990). Eur. J. Mineral. 2, 19-28. Copyright (1990) Schweizerbart, http://www.schweizerbart.de. 
20) but a poor fit to Ising-Glauber, thus showing that ordering schemes can be described by continuous order parameters.

An important consequence of the existence of conserved or partially conserved order parameters is the existence of microstructural solutions to the kinetic equations. Experimental examples of such systems include $\mathrm{O}$-atom vacancy ordering in $\mathrm{YBa}_{2} \mathrm{Cu}_{3} \mathrm{O}_{7}$ superconductors, see Fig. 2 (Schmahl et al., 1989; Krekels et al., 1991) and Al-Si ordering in feldspars and cordierites (Putnis et al., 1987; Wruck et al., 1991; Putnis \& Salje, 1994). The microstructures observed in these materials take the form of 'stripe' or 'tweed' patterns. 'Stripes' consist of more or less regular arrays of parallel domain walls, while 'tweeds' consist of interwoven wall segments. Computer simulations of annealing kinetics using generic models in which elastic degrees of freedom are coupled to chemical ordering develop a tweed pattern initially, which some models show evolving into a stripe pattern on further annealing (Marais, Heine, Nex \& Salje, 1991; Parlinski et al., 1993; Parker et al., 1993; Bratkovsky et al., 1994; Marais et al., 1994). It has been shown that for certain regions of parameter space the one-dimensional form of the time-dependent Landau-theory equations predict stripe phases (Tsatskis et al., 1994).

\section{Conclusions and prospects}

As shown above, phase transitions influence not just the bulk structure of materials but also their microstructure. While we have a good understanding, through techniques such as X-ray diffraction, of bulk domains, our understanding of the structure and properties of interfaces such as twin walls lags behind. Diffraction techniques cannot easily resolve the atomic level structure of interfaces. Simulation studies do offer us a window into these structures, but they typically investigate topologically perfect interfaces in pure crystals, neglecting the chemical degrees of freedom possessed by real crystals.

The most important tool for the interpretation of phasetransition-induced microstructures is Landau theory. Originally, Landau theory was developed to study single domains at temperatures close to the transition temperature (but outside the Ginzburg interval). In fact, simple Landau potentials often provide a good description of the thermodynamics of phase transitions over a wide temperature range. With the addition of the Ginzburg term, Landau theory can describe, on a mesoscopic level, the structure of domain walls and surfaces. Through time-dependent Landau theory, the kinetic equations that lead to tweed and twin wall microstructures can be determined. The results of Landau theory are consistent with the results of experiment and, where experimental data are lacking, with simulation results.

There are many experimental questions that need to be answered. For instance, is the anomalously fast domain wall diffusion of $\mathrm{Na}$ ions and $\mathrm{O}$-atom vacancies seen in $\mathrm{WO}_{3}$ seen in any other materials and do the doped walls in those materials show unusual properties, such as the superconductivity of Na-doped $\mathrm{WO}_{3}$ walls? So far only a handful of systems have been investigated either experimentally or by simulation. Furthermore, the reason why $\mathrm{WO}_{3}$ shows this behaviour is also unknown, although the most reasonable explanation seems to be that there is a large concentration of vacancies within the wall. The answer to this question will probably come from simulation studies, since advances in computing power and simulation methodologies such as the linear scaling code SIESTA (Soler et al., 2002) make possible the atomic level simulation of mesoscopic systems.

It is possible to imagine a large number of technological applications for doped twin walls. It is already possible to engineer twin wall microstructures using techniques such as lithography. For example, doped $\mathrm{WO}_{3}$ twin wall microstructures could be used as superconducting Josephson junctions. Another possibility could be to use twin walls as reaction chambers. Reactants could be diffused in from opposite surfaces intersecting a twin wall. Inside the wall, the reactants would be forced to occupy a fixed orientation by steric effects. Thus the reactants would only come together at a specific orientation, forming products that would not occur in conventional reactions.

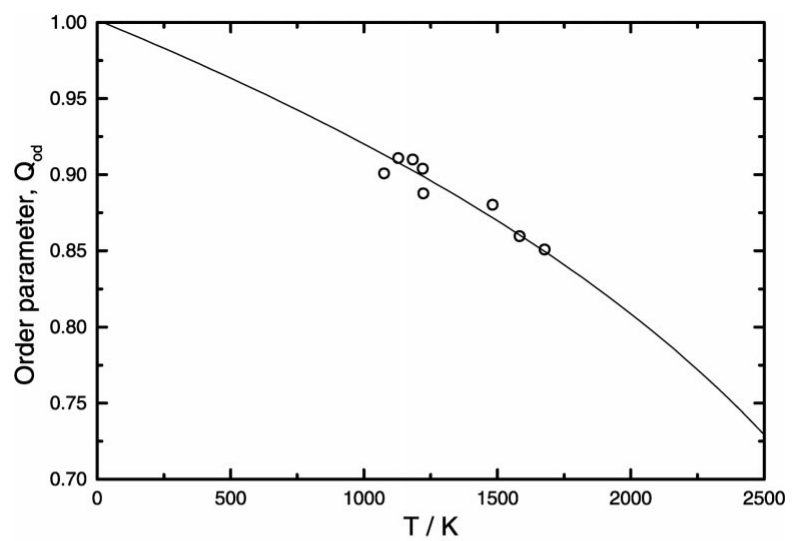

(a)

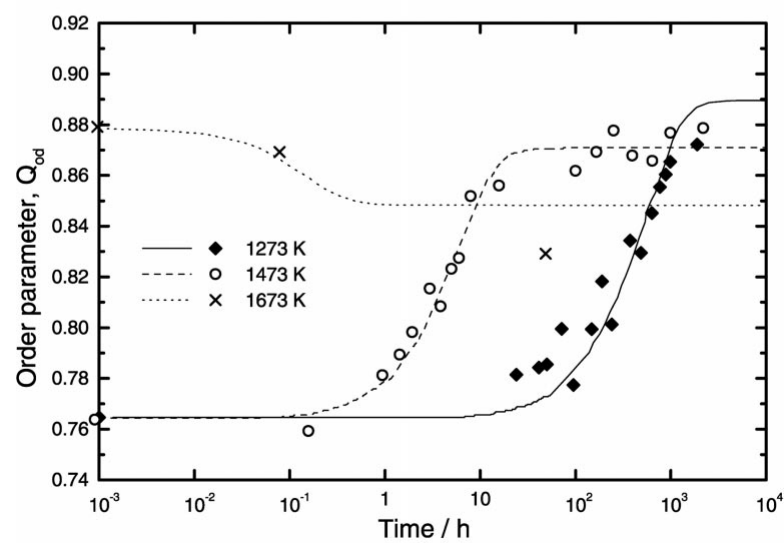

(b)

Figure 20

(Al, Si) order/disorder phase transition in anorthite. These figures show the $(a)$ equilibrium and $(b)$ kinetic behaviours of anorthite fitted using Landau theory. The phase transition is close to tricritical, with $T_{C} \approx$ 3475 K (Salje et al., 1993). 


\section{References}

Ahn, K. H., Lookman, T. \& Bishop, A. R. (2004). Nature (London), 428, 401-404.

Aird, A. \& Salje, E. K. H. (2000). Eur. Phys. J. 15, 205-210.

Bishop, A. R., Lookman, T., Saxena, S. \& Shenoy, S. R. (2003). Europhys. Lett. 63, 289-295.

Bismayer, U., Hensler, J., Salje, E. \& Güttler, B. (1994). Phase Transitions, 48, 149-168.

Bismayer, U., Mathes, D., Bosbach, D., Putnis, A., Van Tendeloo, G., Novak, J. \& Salje, E. K. H. (2000). Mineral. Mag. 64, 233-229.

Bosbach, D., Putnis, A., Bismayer, U. \& Güttler, B. (1997). J. Phys. Condens. Matter, 9, 8397-8405.

Boulestieux, C., Yangui, B., Nihoul, G. \& Barret, A. (1983). $J$. Microsc. 129, 315-326.

Bourim, E. M., Tanaka, H., Gabbay, M., Fantozzi, G. \& Cheng, B. L. (2002). J. Appl. Phys. 91, 6662-6669.

Bratkovsky, A. M., Salje, E. K. H., Marais, S. C. \& Heine, V. (1994). Phase Transitions, 48, 1-13.

Bruce, A. D. (1980). Adv. Phys. 29, 111-217.

Bussmann-Holder, A., Dalal, N., Fu, R. \& Migoni, R. (2001). J. Phys. Condens. Matter, 13, L231-L237.

Cahn, J. W. (1961). Acta Metal. 9, 795-801.

Cahn, J. W. (1962). Acta Metal. 10, 179-183.

Cahn, J. W. (1968). Trans. Am. Inst. Metall. Eng. 242, 166-180.

Calleja, M., Dove, M. \& Salje, E. K. H. (2001). J. Phys. Condens. Matter, 13, 9445-9454.

Calleja, M., Dove, M. \& Salje, E. K. H. (2003). J. Phys. Condens. Matter, 15, 2301-2307.

Cámara, F., Carpenter, M. A., Domeneghetti, M. C. \& Tazzoli, V. (2003). Am. Mineral. 88, 1115-1128.

Cámara, F., Doukhan, J. C. \& Salje, E. K. H. (2000). Phase Transitions, 71, 227-242.

Carpenter, M. A. (1992). Phys. Chem. Miner. 19, 1-24.

Carpenter, M. A. (2000). Rev. Mineral. Geochem. 39, 35-64.

Carpenter, M. A., Domeneghetti, M.-C. \& Tazzoli, V. (1990a). Eur. J. Mineral. 2, 7-18.

Carpenter, M. A., Domeneghetti, M.-C. \& Tazzoli, V. (1990b). Eur. J. Mineral. 2, 19-28.

Carpenter, M. A. \& Salje, E. K. H. (1989). Mineral. Mag. 53, 483-501.

Carpenter, M. A., Salje, E. K. H. \& Graeme-Barber, A. (1998). Eur. J. Mineral. 10, 621-692.

Carpenter, M. A., Salje, E. K. H., Graeme-Barber, A., Wruck, B., Dove, M. T. \& Knight, K. S. (1988). Am. Mineral. 83, 2-22.

Chihara, H., Nakamura, N. \& Tachiki, M. (1973). J. Chem. Phys. 59, 5387-5391.

Chrosch, J. \& Salje, E. K. H. (1994). Physica (Utrecht), C225, 111-116.

Chrosch, J. \& Salje, E. K. H. (1999). J. Appl. Phys. 85, 722-727.

Conti, S. \& Salje, E. K. H. (2001). J. Phys. Condens. Matter, 13, L847-L854.

Dalal, N., Klymachyov, A. \& Bussmann-Holder, A. (1998). Phys. Rev. Lett. 81, 5924-5927.

Devonshire, A. F. (1949). Philos. Mag. 40, 1040-1063.

Dove, M. T., Giddy, A. P. \& Heine, V. (1992). Ferroelectrics, 136, 33-49.

Fally, M., Kubinec, P., Fuith, A., Warhanek, H. \& Filipic, C. (1995). J. Phys. Condens. Matter, 7, 2195-2204.

Frondel, C. (1945). Am. Mineral. 30, 447-68.

Fujishita, H., Ishikawa, Y., Tanaka, S., Ogawaguchi, A. \& Katano, S. (2003). J. Phys. Soc. Jpn, 72, 1426-1435.

Gay, D. H. \& Rohl, A. L. (1995). J. Chem. Soc. Faraday Trans. 91, 925-936.

Goto, T. \& Lüthi, B. (2003). Adv. Phys. 52, 67-118.

Glauber, R. J. (1963). J. Math. Phys. 4, 294-307.

Harrison, R. J. \& Redfern, S. A. T. (2002). Phys. Earth Planet. Inter. 134, 253-272.

Harrison, R. J., Redfern, S. A. T., Buckley, A. \& Salje, E. K. H. (2004). J. Appl. Phys. 95, 1706-1717.
Harrison, R. J., Redfern, S. A. T. \& Street, J. (2003). Am. Mineral. 88, 574-582.

Harrison, R. J. \& Salje, E. K. H. (1994). Phys. Chem Miner. 21, 325-329.

Hartman, P. (1989). J. Cryst. Growth, 96, 667-672.

Hartman, P. \& Bennema, P. (1980). J. Cryst. Growth 49, 145-156.

Hayward, S. A., Chrosch, J., Salje, E. K. H. \& Carpenter, M. A. (1996). Eur J. Mineral. 8, 1301-1310.

Hayward, S. A. \& Salje, E. K. H. (1996). Am. Mineral. 81, 1332-1336.

Hayward, S. A. \& Salje, E. K. H. (2000). Mineral. Mag. 64, 195-200.

Heaney, P. J. \& Veblen, D. R. (1991). Am. Mineral. 76, 1459-66.

Hill, N. A. \& Filippeti, A. (2002). J. Magn. Magn. Mater. 242, 976-979.

Hilliard, J. E. (1970). Phase Transformations, edited by H. I. Anderson, pp. 497-560. Washington, DC: American Society for Metals.

Houchmandzadeh, B., Lajerowicz, J. \& Salje, E. K. H. (1992). J. Phys. Condens. Matter, 4, 9779-9794.

Huang, Y. N., Li, X., Ding, Y., Wang, Y. N., Shen, H. M., Zhang, Z. F., Fang, C. S., Zhuo, S. H. \& Fung, P. C. W. (1997). Phys. Rev. B, 55, 16159-16167.

Hur, N., Park, S., Sharma, P. A., Ahn, J. S., Guha, S. \& Cheong, S.-W. (2004). Nature (London), 429, 392-395.

Imry, Y. (2001). Introduction to Mesoscopic Physics, 2nd ed. Oxford University Press.

Kawasaki, K. (1966). Phys. Rev. 145, 224-230.

Kityk, A. V., Schranz, W., Sondergeld, P., Havlik, D., Salje, E. K. H. \& Scott, J. F. (2000). Phys. Rev. B, 61, 946-956.

Krekels, T., Van Tendeloo, G., Broddin, D., Amelinkx, S., Tanner, L., Mehbod, M., Vanlanthem, E. \& Deltour, R. (1991). Physica (Utrecht), C173, 361-376.

Lagraff, J. R. \& Payne, D. A. (1992). Ferroelectrics, 130, 87-105.

LaFemina, J. P. (1992). Surf. Sci. Rep. 16, 137-260.

Landau, L. D. (1937). Zh. Eksp. Teor. Fiz. 7, 19ff. Translated (1965) in Collected Papers of L. D. Landau, by D. Ter Harr, pp. 193-209. Oxford: Pergamon Press.

Langer, J. S. (1971). Ann. Phys. (Leipzig), 65, 53-83.

Lee, W. T., Dove, M. T. \& Salje, E. K. H. (2000). J. Phys. Condens. Matter, 12, 9829-9841.

Lee, W. T. \& Salje, E. K. H. (2000). Eur. Phys. J. B13, 395-398.

Lee, W. T., Salje, E. K. H. \& Bismayer, U. (2002). J. Phys. Condens. Matter, 14, 7901-7910.

Lee, W. T., Salje, E. K. H. \& Bismayer, U. (2003). J. Phys. Condens. Matter, 15, 1353-1366.

Lee, W. T., Salje, E. K. H. \& Dove, M. T. (1999). J. Phys. Condens. Matter, 11, 7385-7410.

Leeuw, N. H. de \& Parker, S. C. (1997). J. Chem. Soc. Faraday Trans. 93, 467-476.

Lehnert, H., Boysen, H., Frey, F., Hewat, A. \& Radaelli, P. (1997). Z. Kristallogr. 212, 712-719.

Lifshitz, E. M. \& Pitaevskii, L. P. (1981). Physical Kinetics. Oxford: Butterworth-Heinemann.

Locherer, K. R., Chrosch, J. \& Salje, E. K. H. (1998). Phase Transitions, 67, 51-63.

Lüth, H. (1993). Surfaces and Interfaces of Solids. Berlin: SpringerVerlag.

Mackrodt, W. C., Davey, R. J., Black, S. N. \& Docherty, R. (1987). J. Cryst. Growth, 80, 441-446.

Marais, S., Heine, V., Nex, C. \& Salje, E. K. H. (1991). Phys. Rev. Lett. 66, 2468-2483.

Marais, S., Heine, V. \& Salje, E. K. H. (1991). Phys. Chem. Miner. 18, 180-183.

Marais, S. \& Salje, E. K. H. (1991). J. Phys. Condens. Matter, 3, 3667-3670.

Marais, S. C., Salje, E. K. H., Heine, V. \& Bratkovsky, A. M. (1994). Phase Transitions, 48, 15-45.

Meitu, H., Kitahara, K. \& Ross, J. (1976a). J. Chem. Phys. 64, 292-299. 
Meitu, H., Kitahara, K. \& Ross, J. (1976b). J. Chem. Phys. 65, 363-396.

Millis, A. J. (1998). Nature (London), 392, 147-150.

Novak, J., Bismayer, U. \& Salje, E. K. H. (2002). J. Phys. Condens. Matter, 14, 657-664.

Novak, J. \& Salje, E. K. H. (1998). J. Phys. Condens. Matter, 10, L359-L366.

Parker, S. C., Kelsey, E. T., Oliver, P. M. \& Titiloye, J. O. (1993). Faraday Discuss. 95, 75-84.

Parlinski, K., Salje, E. K. H. \& Heine, V. (1993). Acta Metall. Mater. 41, 839-847.

Parsons, I., Brown, W. L. \& Smith, J. V. (1999). Contrib. Mineral. Petrol. 136, 92-110.

Pérez-Mato, J. M. \& Salje, E. K. H. (2000). J. Phys. Condens. Matter, 12, L29-L34.

Pertsev, N. A., Novak, J. \& Salje, E. K. H. (2000). Philos. Mag. A80, 2201-2213.

Philips, R. (2001). Crystals, Defects and Microstructures. Cambridge University Press.

Phillips, B. L., McGuinn, M. D. \& Redfern, S. A. T. (1997). Am. Mineral. 82, 1-7.

Putnis, A. \& Salje, E. K. H. (1994). Phase Transitions, 48, 85-105.

Putnis, A., Salje, E. K. H., Redfern, S. A. T., Fyfe, C. A. \& Stroble, H. (1987). Phys. Chem. Miner. 14, 446-454.

Radescu, S., Etxebarria, I. \& Pérez-Mato, J. M. (1995). J. Phys. Condens. Matter, 7, 585-595.

Rae, A. I. M. (1982). J. Phys. C15, 1883-1896.

Redfern, S. A. T. (1998). Physica (Utrecht), B241-243, 1189-1196.

Redfern, S. A. T., Artioli, G., Rinaldi, R., Henderson, C. M. B., Knight, K. S. \& Wood, B. J. (2000). Phys. Chem. Miner. 27, 630-637.

Redfern, S. A. T. \& Sartbaeva, A. (2002). Geochim. Cosmochim. Acta, 66, A627.

Roewer, R. W., Bismayer, U., Morgenroth, W. \& Güttler, B. (1997). Solid State Ionics, 101-103, 585-589.

Roucau, C., Tanaka, M., Torres, J. \& Ayroles, R. (1979). J. Microsc. 4, 603-610.

Salje, E. K. H. (1988). Phys. Chem Miner. 15, 336-348.

Salje, E. K. H. (1990). Phase Transitions in Ferroelastic and Co-elastic Crystals. Cambridge University Press.

Salje, E. K. H. (2000). Rev. Mineral. Geochem. 39, 65-84.

Salje, E. K. H., Bismayer, U., Hayward, S. A. \& Novak, J. (2000). Mineral. Mag. 64, 201-212.

Salje, E. \& Devarajan, V. (1986). Phase Transitions, 6, 235-248.

Salje, E. K. H. \& Ishibashi, Y. (1996). J. Phys. Condens. Matter, 8, 8477-8495.

Salje, E. K. H., Kuscholke, B., Wruck, B. \& Kroll, H. (1985). Phys. Chem. Mineral. 12, 99-107.

Salje, E. K. H. \& Marais, S. (1992). Ferroelectrics, 136, 1-13.

Salje, E. K. H., Wruck, B., Graeme-Barber, A. \& Carpenter, M. A. (1993). J. Phys. Condens. Matter, 5, 2961-2968.
Salje, E. K. H., Wruck, B. \& Thomas, H. (1991). Z. Phys. B82, 399-404.

Schmahl, W. W., Putnis, A., Salje, E., Freeman, P., Graeme-Barber, A., Jones, R., Singh, K. K., Blunt, J., Edwards, P. P., Loram, J. \& Mirza, K. (1989). Philos. Mag. Lett. 60, 241-248.

Schmid, H. (1994). Ferroelectrics, 162, 317-338.

Schranz, W. (1997). Phase Transitions, 64, 103-114.

Schranz, W., Troster, A., Kityk, A. V., Sondergeld, P. \& Salje, E. K. H. (2003). Europhys. Lett. 62, 512-518.

Snoeck, E., Normand, L., Thorel, A. \& Roucau, C. (1994). Phase Transitions, 46, 77-88.

Soler, J.-M., Artacho, E., Gale, J. D., García, A., Junquera, J., Ordejón, P. \& Sánchez-Portal, D. (2002). J. Phys. Condens. Matter, 14, 2745-2779.

Stokes, H. T. \& Hatch, D. M. (1988). Isotropy Subgroups of the 230 Crystallographic Space Groups. Singapore: World Scientific.

Strukov, B. A. \& Levanyuk, A. (1998). Ferroelectric Phenomena in Crystals: Physical Foundations. Berlin: Springer-Verlag.

Titiloye, J. O., Parker, S. C. \& Mann, S. (1993). J. Cryst. Growth, 131, 533-545.

Tsai, F., Khiznichenko, V. \& Cowley, J. M. (1992). Ultramicroscopy, 45, 55-63.

Tsatskis, I., Salje, E. K. H. \& Heine, V. (1994). J. Phys. Condens. Matter, 6, 11027-11034.

Voronkova, V. I. \& Wolf, T. (1993). Physica (Utrecht), C218, 175-180.

Whittborn, J., Canallas, C., Rao, K. V., Clemens, R., Karlsson, H. \& Laurell, F. (2002). Appl. Phys. Lett. 80, 1622-1624.

Wood, V. E. \& Austin, A. E. (1975). Magnetoelectric Interaction Phenomena in Crystals, edited by A. J. Freeman \& H. Schmid, pp. 181-194. New York: Gordon and Breach.

Wruck, B., Salje, E. K. H. \& Graeme-Barber, A. (1991). Phys. Chem. Miner. 17, 700-710.

Wruck, B., Salje, E. K. H., Zhang, M., Abraham, T. \& Bismayer, U. (1994). Phase Transitions, 48, 135-148.

Wulff, G. (1901). Z. Kryst. Mineral. 34, 449-531.

Xu, H. W. \& Heaney, P. J. (1997). Am. Mineral. 82, 99-108.

Yamamoto, M., Yagi, K. \& Honjo, G. (1977). Phys. Status Solidi A, 41, 523-34.

Yau, S.-T. \& Vekilov, P. G. (2000). Nature (London), 406, 494-497.

Zalar, B., Laguta, V. V. \& Blinc, R. (2003). Phys. Rev. Lett. 90, 037601.

Zangwill, A. (1988). Physics at Surfaces. Cambridge University Press.

Zapart, W. (2003). Ferroelectrics, 70, 1077-1094.

Zhao, Y., Weidner, D. J., Parise, J. B. \& Cox, D. E. (1993). Phys. Earth Planet. Inter. 76, 17-34.

Zheng, H., Wang, J., Lofland, S. E., Ma, Z., Mohaddes-Ardabili, L., Zhao, T., Salamanca-Riba, L., Shinde, S. R., Ogale, S. B., Bai, F., Viehland, D., Jia, Y., Schlom, D. G., Wuttig, M., Roytburd, A. \& Ramesh, R. (2004). Science, 303, 661-663.

Zimmermann, M. \& Schranz, W. (1996). J. Phys. Condens. Matter, 8, $7085-7103$. 